\title{
1 A new lineage of non-photosynthetic green algae with extreme organellar genomes
}

3 Tomáš Pánek ${ }^{1,2}$, Dovilè Barcyte ${ }^{1}$, Sebastian C. Treitli ${ }^{3}$, Kristína Záhonová $^{1}$, Martin Sokol $^{1}$, Tereza

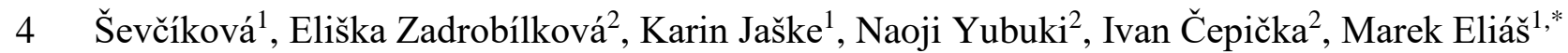

$6{ }^{1}$ Department of Biology and Ecology, Faculty of Science, University of Ostrava, 70100 Ostrava,

7 Czech Republic

$8{ }^{2}$ Department of Zoology, Faculty of Science, Charles University, 12843 Prague, Czech Republic

$9{ }^{3}$ Department of Parasitology, Faculty of Science, Charles University, BIOCEV, 25242 Vestec,

10 Czech Republic

11

12 Correspondence: marek.elias@osu.cz

13

14

15

16 


\section{Abstract}

25 Background: The plastid genomes of the green algal order Chlamydomonadales tend to expand

26 their non-coding regions, but this phenomenon is poorly understood. Here we shed new light on

27 organellar genome evolution in Chlamydomonadales by studying a previously unknown non-

28 photosynthetic lineage. We established cultures of two new Polytoma-like flagellates, defined their

29 basic characteristics and phylogenetic position, and obtained complete organellar genome

30 sequences and a transcriptome assembly for one of them.

31 Results: We discovered a novel deeply diverged chlamydomonadalean lineage that has no close

32 photosynthetic relatives and represents an independent case of photosynthesis loss. To

33 accommodate these organisms, we establish a new genus, Leontynka, with two species L. pallida

34 and L. elongata distinguished by both morphological and molecular characteristics. Notable

35 features of the colourless plastid of L. pallida deduced from the plastid genome (plastome)

36 sequence and transcriptome assembly include the retention of ATP synthase, thylakoid-associated

37 proteins, carotenoid biosynthesis pathway, and plastoquinone-based electron transport chain, the

38 latter two modules having an obvious functional link to the eyespot present in Leontynka. Most

39 strikingly, the L. pallida plastome with its $\sim 362 \mathrm{kbp}$ is by far the largest among non-photosynthetic

40 eukaryotes investigated to date. Instead of a high gene content, its size reflects extreme

41 proliferation of sequence repeats. These are present also in coding sequences, with one repeat type

42 found in exons of 11 out of 34 protein-coding genes and up to 36 copies per gene, affecting thus

43 the encoded proteins. The mitochondrial genome of L. pallida is likewise exceptionally large, with

44 its >104 kbp surpassed only by the mitogenome of Haematococcus lacustris among all members

45 of Chlamydomonadales studied so far. It is also bloated with repeats, yet completely different from

46 those in the L. pallida plastome, which contrasts with the situation in H. lacustris where both 
47 organellar genomes have accumulated related repeats. Furthermore, the L. pallida mitogenome

48 exhibits an extremely high GC content in both coding and non-coding regions and, strikingly, a

49 high number of predicted G-quadruplexes.

50 Conclusions: With the unprecedented combination of plastid and mitochondrial genome

51 characteristics, Leontynka pushes the frontiers of organellar genome diversity and becomes an

52 interesting model for studying organellar genome evolution.

53 Keywords: Chlamydomonadales; GC content; G-quadruplex; green algae; mitochondrial genome;

54 non-photosynthetic algae; plastid genome; repeat expansion

\section{Background}

57 Secondary loss of photosynthesis has occurred numerous times across the diversity of plastid-

58 bearing eukaryotes, including land plants (Hadariová et al., 2018; Sibbald \& Archibald, 2020).

59 Among algae, photosynthesis loss has been most common among groups characterised by

60 secondary or higher-order plastids, with chrysophytes and myzozoans (including apicomplexans

61 as the best-studied non-photosynthetic "algae") being the most prominent examples. In green

62 algae, loss of photosynthesis is restricted to several lineages within two classes, Trebouxiophyceae

63 and Chlorophyceae (Figueroa-Martinez et al., 2015). Colourless trebouxiophytes are formally

64 classified in two genera, Helicosporidium and the polyphyletic Prototheca, collectively

65 representing three independent photosynthesis loss events (Suzuki et al., 2018). While these

66 organisms live as facultative or obligate parasites of metazoans (including humans), non-

67 photosynthetic members of Chlorophyceae are all free-living osmotrophic flagellates. Two genera

68 of such colourless flagellates have been more extensively studied and are represented in DNA

69 sequence databases: the biflagellate Polytoma and the tetraflagellate Polytomella. They both fall 
70 within the order Chlamydomonadales (Volvocales sensu lato), but are not closely related to each

71 other. Furthermore, Polytoma as presently circumscribed is polyphyletic, since P. oviforme does

72 not group with its congeners, including the type species P. uvella (Figueroa-Martinez et al., 2015).

73 Hence, photosynthesis was lost at least three times in Chlamydomonadales, but the real number is

74 probably higher, since several other genera of colourless flagellates morphologically falling within

75 this group were historically described (Ettl, 1983), but remain to be studied by modern methods.

76 Indeed, a taxonomically unidentified non-photosynthetic chlamydomonadalean (strain NrC1902),

77 not related to any of the three known lineages, was reported recently (Kayama et al., 2020);

78 whether it corresponds to any of the previously formally described taxa is yet to be investigated.

The non-photosynthetic chlamydomonadaleans are not only diverse phylogenetically, but

80 they also exhibit diversity in the features of their residual plastids. Most notably, Polytomella

81 represents one of the few known cases of a complete loss of the plastid genome (plastome) in a

82 plastid-bearing eukaryote (Smith \& Lee, 2014). In contrast, Polytoma uvella harbours the largest

83 plastome amongst all non-photosynthetic eukaryotes studied to date ( $\geq 230 \mathrm{kbp})$. This is not due to

84 preserving a large number of genes, but because of the massive accumulation of long arrays of

85 short repeats in intergenic regions (Figueroa-Martinez et al., 2017). The unusual architecture of

86 the $P$. uvella plastome seems to reflect a more general trend of plastome evolution in

87 Chlamydomonadales, i.e. a tendency to increase in size by the expansion of repetitive sequences.

88 An extreme manifestation of this trend was recently unveiled by sequencing the $1.35-\mathrm{Mbp}$

89 plastome of the photosynthetic species Haematococcus lacustris, a record-holder amongst all fully

90 sequenced plastomes to date (Bauman et al., 2018; Smith, 2018). Interestingly, H. lacustris also

91 harbours the by far largest known mitochondrial genome (mitogenome) amongst all

92 Chlamydomonadales, which has expanded to $126.4 \mathrm{kbp}$ by the accumulation of repeats highly 
93 similar to those found in the plastome, suggesting an inter-organellar transfer of the repeats (Zhang

94 et al., 2019). The mechanistic underpinnings of the repeat accumulation in chlamydomonadalean

95 organellar genomes are still not clear.

96 When studying protists living in hypoxic sediments, we obtained cultures of two colourless

97 flagellates that turned out to represent a novel, deeply separated lineage of Chlamydomonadales.

98 We here describe them formally as two species in a new genus. Using a combination of different

99 DNA sequencing technologies, we determined sequences of organellar genomes of one of the

100 isolates, which turned out to exhibit extreme features concerning the size and/or composition. Our

101 analysis of these genomes provides important new insights into the evolution of organelle genomes

102 in general.

103

104 Results

\section{A new lineage of non-photosynthetic Chlamydomonadales with two species}

106 Based on their 18S rRNA gene sequences, the two new isolates - AMAZONIE and MBURUCU

107 - constitute a clade (with full bootstrap support) that is nested within Chlamydomonadales, but

108 separate from all the principal chlamydomonadalean clades as demarcated by Nakada et al. (2008)

109 (Fig. 1a). Notably, this new lineage is clearly unrelated to all previously studied non-

110 photosynthetic chlamydomonadaleans, including Polytomella (branching off within the clade

111 Reinhardtinia), both lineages representing the polyphyletic genus Polytoma (P. uvella plus several

112 other species in the clade Caudivolvoxa and $P$. oviforme in the clade Xenovolvoxa), and the strain

$113 \mathrm{NrCl902}$ (also in Caudivolvoxa; Additional file 1: Fig. S1). Our two strains were mutually

114 separated in the 18S rRNA gene tree as deeply as other chlamydomonadalean pairs classified as

115 separate species or even genera, and their 18S rRNA gene sequences differed in 13 positions (out 
116 of 1703 available for comparison). In addition, the ITS1-5.8S-ITS2 rDNA regions of the two

117 strains exhibited only $88 \%$ identity and the differences included several compensatory base

118 changes (CBCs) in the helix II of the characteristic secondary structure of the ITS2 region

119 (Additional file 1: Fig. S2). This and morphology-based evidence presented below led us to

120 conclude that the two strains represent two different species of a new genus of

121 chlamydomonadalean algae, which we propose be called Leontynka pallida (strain AMAZONIE)

122 and Leontynka elongata (strain MBURUCU). Formal descriptions of the new taxa are provided in

123 Additional file 2: Note S1.

124 The phylogenetic position of L. pallida was also studied by using protein sequences

125 encoded by its plastome (see below). Phylogenomic analysis of a concatenated dataset of 24

126 conserved proteins encoded by plastomes of diverse members of Chlorophyceae, including a

127 comprehensive sample of available data from Chlamydomonadales, revealed L. pallida as a

128 separate lineage potentially sister to a fully supported broader clade comprising representatives of

129 the clades Caudivolvoxa and Xenovolvoxa (sensu Nakada et al., 2008; Fig. 1b). This position of $L$.

130 pallida received moderate support in the maximum likelihood analysis (nonparametric bootstrap

131 value of 78\%), but inconclusive support from the PhyloBayes analysis (posterior probability of

132 0.68). Importantly, L. pallida was unrelated to Polytoma uvella (nested within Caudivolvoxa with

133 full support). Polytoma oviforme and the genus Polytomella were missing from the analysis due to

134 lack of plastome data or the complete absence of the plastome, respectively.

135 Both Leontynka species lacked a green plastid (chloroplast). Instead, their cells were

136 occupied by a colourless leucoplast containing starch grains, typically filling most of its volume

137 (Fig. 2, Additional file 1: Figs S4 and S5). Two anterior, isokont flagella approximately as long as

138 the cell body emerged from a keel-shaped papilla (Additional file 1: Fig. S3c, d). Cells of both 
139 species also contained two apical contractile vacuoles (Fig. 2c, f, Additional file 1: Fig. S3a, c,

140 Fig. S4a, d, h), a central or slightly posterior nucleus (Additional file 1: Fig. S3c, Fig. S4h),

141 inclusions of yellowish lipid droplets (Fig. 2h, Additional file 1: Fig. S4h), and one or occasionally

142 two eyespots (Fig. 2a, b, and f-j, Additional file 1: Fig. S3a, b, e, g, h, Fig. S4a, c-f, h).

143 Reproduction occurred asexually through zoospore formation, typically with up to four zoospores

144 formed per the mother cell (Additional file 1: Fig. S3g, h). The two species differed in the cell

145 shape and position of the eyespot, as described in more detail in Additional file 2: Note S2.

146 The plastid of both species was bounded by a double membrane and composed of

147 numerous separate compartments connected by narrow "bridges" (Fig. 2d, e, i, j, Additional file

148 1: Fig. S5a-c, h). Each compartment contained either a single large or two smaller starch grains,

149 leaving essentially no room for the stroma or thylakoids. Rarely, starch-free compartments

150 containing membranous inclusions were present (Fig. 2e). The eyespot globules were inside the

151 plastid and were associated with structures that we interpret as thylakoids (Fig. 2j). Mitochondria

152 were highly abundant and contained numerous cristae (Additional file 1: Fig. S5e, f, i). It was

153 impossible to unambiguously determine the crista morphology in L. pallida (Additional file 1: Fig.

154 S5i), but in L. elongata, the cristae were of the discoidal morphotype (Additional file 1: Fig. S5e).

155 Further details on the ultrastructure of Leontynka spp. are presented in Additional file 2: Note S2.

157 The extremely bloated plastome of Leontynka pallida

158 A complete plastome sequence was assembled for L. pallida using a combination of Oxford

159 Nanopore and Illumina reads. It corresponds to a circular-mapping molecule comprising 362307

160 bp (Fig. 3a). Thirty-four protein-coding genes (including two intronic ORFs), 26 tRNA genes (a

161 standard set presumably allowing for translation of all sense codons), and genes for the three 
162 standard rRNAs were identified and annotated in the genome. Three genes are interrupted by

163 introns: atpA with one group I intron that contains an ORF encoding a LAGLIDADG homing

164 endonuclease, tufA with one group II intron that contains an ORF encoding a reverse

165 transcriptase/maturase protein, and $r n l$ with one group I and one group II intron, neither containing

166 an ORF. No putative pseudogenes or apparent gene remnants were identified in the L. pallida

167 plastome.

No genes that encode proteins directly associated with photosynthetic electron transport

169 components and $\mathrm{CO}_{2}$ fixation were identified in the L. pallida plastome. The genes retained encode

170 proteins involved in transcription (RNA polymerase subunits), translation (tufA and ribosomal

171 subunit genes), protein turnover ( $c l p P, f t s H)$, and a protein of an unclear function ( $y c f 1)$. Nearly

172 all these genes have been preserved also in the plastome of $P$. uvella (Figueroa-Martinez et al.,

173 2017), except for rps2. The two non-photosynthetic Leontynka species share the absence of genes

174 for two ribosomal proteins: $r p l 32$, which is, however, also missing from a subset of photosynthetic

175 representatives of Chlamydomonadales, and rpl23 conserved in plastomes of all photosynthetic

176 chlorophytes investigated to date (Turmel \& Lemieux, 2018). Whereas an Rpl32 protein with a

177 predicted plastid-targeting presequence is encoded by the L. pallida nuclear genome (Additional

178 file 3: Table S1), the loss of rpl23 does not seem to be compensated in a similar way. Interestingly,

179 rpl23 has been independently lost also from the plastome of Helicosporidium sp. (Figueroa-

180 Martinez et al., 2017), which suggests that this ribosomal subunit may become dispensable upon

181 the loss of photosynthesis. In contrast to $P$. uvella, the L. pallida plastome has kept the same set

182 of genes encoding ATP synthase subunits as typical for photosynthetic green algae, i.e., atpA,

$183 a t p B, a t p E, a t p F, a t p H$, and $a t p I$. As evidenced by the transcriptome data, the three missing ATP 
184 synthase subunits (AtpC, AtpD, and AtpG) are encoded by the L. pallida nuclear genome and bear

185 predicted plastid-targeting signals (Additional file 3: Table S1).

What makes the L. pallida plastome truly peculiar are the intergenic regions. Their average

187 length is $4.7 \mathrm{kbp}$, which is 1.5 and five times more than the average length of the intergenic regions

188 in the plastomes of $P$. uvella and C. reinhardtii, respectively (Table 1). Furthermore, while the GC

189 content of the $P$. uvella intergenic regions is vastly different from that of coding regions (19\%

190 versus 40\%), the GC content of these two plastome partitions are highly similar in L. pallida (as

191 well as in C. reinhardtii; Table 1). A self-similarity plot generated for the L. pallida plastome

192 revealed a massive repetitiveness of the DNA sequence, with only short islands of unique

193 sequences scattered in the sea of repeats (Fig. 3b). The repeats are highly organised and occur in

194 various arrangements: tandem repeats, interspersed repeats, inverted repeats (palindromes), and

195 other higher-order composite repeated units. As an example, let us take the most abundant repeat,

196 the imperfect palindrome (IP) CAAACCAGT|NN|ACTGGTTAG. It is present in more than 1300

197 copies, with the dinucleotide AA as a predominant form of the internal spacer. This repeat is mostly

198 localised in clusters (>1200 cases) where its copies are interleaved by a repeat with the conserved

199 sequence TAACTAAACTTC, so together they constitute a composite extremely abundant tandem

200 repeat. In a single region, the palindromic repeat combines with a different interspersed repeat

201 (TAACTACTT), together forming a small cluster of composite tandem repeats in 14 copies.

202 Besides, the same palindromic repeat is also part of another, 146 bp-long repeat present in 27

203 copies across the plastome (for details see Additional file 2: Note S3).

204 Apart from intergenic regions, sequence repeats are found also in the four introns present

205 in the L. pallida plastome. The most prominent is a cluster of 20 copies of the motif

206 TGGTTAGTAACTAAACTTCCAAACCAGTAAAC in the intron inside the atpA gene that is 
207 abundant also in intergenic regions (more than 1,000 copies typically located in huge clusters).

208 Strikingly, when analysing the distribution of the most abundant IPs, we noticed that the motif

209 AAGCCAGC|NNN|GCTGACTT and its variants are present also in coding regions, namely in

210 exons of 11 out of 34 protein-coding genes of L. pallida plastome (Fig. 4a-c). They can be present

211 in up to 36 copies per gene ("variant 8" in exons of rpoC2; Fig. 4b). In most cases the IP motif is

212 part of a longer repeat unit including extra nucleotides at both ends ("variant 4" to "variant 8" in

213 Fig. 4c). The most complex repeat unit variant is the following one (the IP core in round brackets;

214 square brackets indicate alternative nucleotides occurring at the same position): AAAGAT-

215 (AAGTCAGC|AGA|GCTGAC[AT]T)-

216 CCAGACCACTAAAGTGGTCAGTAACTAAAAGTTAT. It is restricted to coding sequences

217 (i.e., is absent from intergenic regions and introns) and occurs in eight copies inside three genes

218 (rpoC2, rpoC1, fts $H)$, resulting in an insertion of a stretch of 20 amino acid residues in the encoded

219 proteins. Other repeat variants (listed in Fig. 4c) have proliferated in exons as well as intergenic

220 regions and introns. However, none of the aforementioned nucleotide motifs were found in

221 plastomes of other chlamydomonadalean algae, indicating they have originated and diverged only

222 in the Leontynka lineage.

223 Manual inspection of protein sequence alignments including chlamydomonadalean

224 orthologs of the L. pallida proteins revealed that the intraexonic repeat insertions are located

225 mostly in poorly conserved regions (see Fig. 4d for an example). Preferential proliferation in

226 variable parts of coding regions is consistent with a high abundance of these motifs in proteins that

227 exhibit a general tendency for including rapidly evolving and poorly conserved regions, namely

228 FtsH (Additional file 1: Fig. S6), Ycf1, RpoC1, RpoC2, and RpoBb. Interestingly, the phase and

229 orientation of the intraexonic repeats with respect to the reading frame and the direction of 
230 transcription is not random and is potentially biased such that not only termination codons, but

231 also codons generally rare in L. pallida plastid coding sequences are avoided from the actual frame

232 in which the insertion is read during translation (for details see Fig. 4c, e, Additional file 2: Note

233 S4). This bias does not merely reflect a possible bias in the orientation of the repeats relative to

234 the DNA strand of the genome, as the repeats are distributed roughly equally in both strands when

235 counted at the whole-genome level (Fig. 4c).

237 A high number of potential quadruplex-forming sequences in the GC-rich mitogenome of

\section{Leontynka pallida}

239 The mitogenome sequence was assembled from Nanopore and Illumina reads as a linear molecule

240 of 110515 bp with long ( $5770 \mathrm{bp})$ nearly perfect (97.7\% identity) direct terminal repeats differing

241 primarily by the presence/absence of two short repetitive regions (13 and 70 bp) (Fig. 3c,

242 Additional file 1: Fig. S7). This possibly indicates that the L. pallida mitogenome is in fact circular,

243 with the slight differences in the terminal direct repeats of the assembled linear contigs reflecting

244 sequence variability of a particular genomic region between the different genome copies in $L$.

245 pallida or possibly sequencing or assembly artefacts. If circular, the mitogenome would then have

246 a length of $\sim 104812 \mathrm{bp}$. The suspected circularity of the mitogenome is also compatible with the

247 absence of the $r t l$ gene, which is present in all linear mitogenomes of Chlamydomonadales

248 characterised to date and encodes a reverse transcriptase-like protein implicated in the replication

249 of the mitogenome termini (Smith \& Craig, 2021). Apart from rtl, the gene content of the L. pallida

250 mitogenome is essentially the same as in other chlamydomonadalean mitogenomes sequenced

251 before and includes seven protein-coding genes (with coxl interrupted by an ORF-free group II

252 intron), only three tRNA genes, and regions corresponding to the $16 \mathrm{~S}$ and $23 \mathrm{~S}$ rRNA genes. As in 
other chlamydomonadaleans studied in this regard (Boer \& Gray, 1988; Denovan-Wright et al.,

254 1994; Fan et al., 2003), the mitochondrial 16S and 23S rRNA genes in L. pallida are fragmented,

255 consisting of multiple separately transcribed pieces. Four fragments, together constituting a

256 presumably complete $16 \mathrm{~S}$ rRNA, were annotated by considering the sequence and secondary

257 structure conservation of the molecule. The number of the 16S rRNA fragments is thus the same

258 as in Chlamydomonas reinhardtii, but the breakpoints are not completely identical. Due to a lower

259 conservation of the $23 \mathrm{~S}$ rRNA molecule, we could identify only a few of the presumed gene

260 fragments in the L. pallida mitogenome.

261 The large size and the low density of coding sequences of the L. pallida mitogenome

$262(\sim 84.7 \%$ of its complete sequence is represented by intergenic regions) are atypical for

263 Chlamydomonadales, including the other non-photosynthetic species: the mitogenome of $P$. uvella

264 is $17.4 \mathrm{kbp}$ long (Del Vasto et al., 2015), and in Polytomella spp. the mitogenome size ranges from

$265 \sim 13$ to $24.4 \mathrm{kbp}$ (Smith et al., 2010; Smith et al., 2013). In fact, the L. pallida mitogenome can be

266 compared only to the recently characterised mitogenome of Haematococcus lacustris, which with

267 the same gene content is even larger $(126.4 \mathrm{kbp})$ yet with a similar representation of intergenic

268 regions (83.2\%). A self-similarity plot generated for the L. pallida mitogenome revealed a highly

269 repetitive nature of the genome sequence (Fig. 3d), similar to the plastome. However, the repeats

270 are distributed less evenly than in the plastome, being present particularly in the terminal regions

271 of the assembled linear sequence and in several internal hotspots.

272 With the GC content $62.6 \%$ (as counted for the circularised version of the genome), the

273 mitogenome of L. pallida has the third highest documented mitochondrial GC content out of

274 11,077 examined mitogenomes available in GenBank, being surpassed only by the lycophyte

275 Selaginella moellendorffii (68.2\%; Hecht et al., 2011) and the green alga Picocystis salinarum 
$276(67.7 \%)$. These values contrast sharply with the median GC content value for the whole set of the

277 mitogenomes examined, i.e. 38\%. We also encountered an exceptionally high GC content (63.4\%)

278 and a strong bias towards using GC-rich codons in all protein-coding genes in the L. pallida

279 mitogenome (see Additional file 3: Tables S2 and S3). Only two organisms are presently known

280 to have an even higher GC content of mitochondrial protein-coding genes: the sponge

281 Leucosolenia complicata (71.2\%; Lavrov et al., 2016) and P. salinarum (67.9\%). Some L. pallida

282 mitogenome-encoded proteins, namely $\mathrm{Nad} 2$ and $\mathrm{Nad} 5$, also exhibit a higher relative content of

283 amino acids with GC-rich codons ( $\mathrm{G}, \mathrm{A}, \mathrm{R}$, and $\mathrm{P})$ compared to most of their orthologs in other

284 species (Additional file 3: Table S4). Thus, not only the expanded GC-rich intergenic regions, but

285 also coding regions of the L. pallida mitogenome contribute to its extremely high GC content.

286 The repeats in the plastome and mitogenome of $H$. lacustris are nearly identical (Zhang et

287 al., 2019), so it was interesting to compare the two L. pallida organellar genomes to find out

288 whether they behave similarly. However, as follows from the respective similarity plot (Fig. 3e)

289 and comparison of most abundant inverted repeats and palindromes (Fig. 4a), the repeats in the

290 two genomes do not resemble each other. The proliferation of different repeats in the two

291 organellar genomes of L. pallida at least partially accounts for their strikingly different GC content

292 (62.6\% vs 37\%). Interestingly, the most abundant IP in the L. pallida mitogenome contains the

293 GGGG motif (Fig. 4a), which prompted us to bioinformatically investigate the possible occurrence

294 of G-quadruplexes, unusual secondary structures in nucleic acids formed by guanine-rich regions

295 (Burge et al., 2006). Indeed, the L. pallida mitogenome was suggested to include up to 14.7

296 potential quadruplex-forming sequences (PQS) per 1,000 bp. A similar value was inferred for the

297 S. moellendorffii mitogenome (15.6 PQS per 1,000 bp), whereas the other mitochondrial and

298 plastid genomes that we analysed for comparison (for technical reasons focusing on GC-rich 
genomes only) exhibited a much lower values (0.0-6.9 PQS per 1,000 bp; see Additional file 3:

300 Table S2).

\section{Discussion}

303 Both 18S rRNA and plastid gene sequence data concur on the conclusion that the two strains

304 investigated in this study, AMAZONIE and MBURUCU, represent a phylogenetically novel

305 lineage within Chlamydomonadales that is unrelated to any of the previously known non-

306 photosynthetic lineages in this order, i.e. Polytomella, Polytoma sensu stricto (including the type

307 species P. uvella), Polytoma oviforme, and the recently reported strain NrC1902. However,

308 morphological features of AMAZONIE and MBURUCU, including the cell shape and the

309 presence of two flagella, papilla, eyespot, and starch granules, make our organisms highly

310 reminiscent of the genus Polytoma (Ettl, 1983). This is consistent with the previous insight that

311 the Polytoma morphotype does not define a coherent phylogenetic unit (Figueroa-Martinez et al.,

312 2015). All other historically described genera of colourless flagellates assigned formerly to

313 Chlamydomonadales are sufficiently different from our strains as to consider them a potential

314 taxonomic home for AMAZONIE and MBURUCU (see Additional file 2: Note S5), justifying the

315 erection of the new genus Leontynka to accommodate the two strains. Furthermore, these strains

316 clearly differ from each other in morphology (cell shape and size, position of the eyespot) and are

317 genetically differentiated, as apparent from the comparison of the 18S rRNA gene and ITS2 region

318 sequences. Indeed, given the presence of several CBCs in the helix II of the conserved ITS2

319 secondary structure, the two strains are predicted to be sexually incompatible and hence

320 representing separate "biological species" (Coleman, 2000; Wolf et al., 2013). We considered a

321 possibility that AMAZONIE and MBURUCU may represent some of the previously described 
322 Polytoma species, but as detailed in Additional file 2: Note S5, none seems to be close enough in

323 morphology as reported in the original descriptions. Given the fact that the majority of Polytoma

324 species have been isolated and described from central Europe whereas our strains both come from

325 tropical regions of South America, it is not so surprising that we encountered organisms new to

326 science.

Leontynka spp. exhibit a number of ultrastructural similarities to the previously studied Polytoma species (Lang, 1963; Siu et al., 1976; Gaffal \& Schneider, 1980). For example, although photosynthetic chlamydomonadalean flagellates usually contain only a few mitochondria squeezed between the nucleus and the plastid, the cells of non-photosynthetic taxa, including Leontynka, are mitochondria-rich. It is possible that the proliferation of mitochondria compensates for the loss of the energetic function of the plastid in the non-photosynthetic species. Previous ultrastructural studies of Polytoma obtusum (Siu et al., 1976) and Polytomella sp. (Dudkina et al., 2010) showed that their mitochondria possess lamellar or irregular tubulo-vesicular cristae, respectively. The cristae of $L$. pallida resemble the latter morphotype, whereas L. elongata most probably possesses discoidal cristae (Additional file 1: Fig. S5e, f). Discoidal cristae are a very rare morphotype within the supergroup Archaeplastida, although they apparently evolved several times independently during the eukaryote evolution (Pánek et al., 2020) and were previously noticed in several other non-photosynthetic chlorophytes (Polytoma uvella, Polytomella agilis, and Prototheca zopfii; Webster et al., 1967).

A particularly notable feature of Leontynka spp. is the presence of two eyespots. These

342 were more frequent in L. elongata (about half of the cells had two eyespots), whereas in the $L$. 343 pallida cultures, such cells were rather rare. Variation in the number of eyespots (from none to 344 multiple) in Chlamydomonas reinhardtii was shown to be a result of genetic mutations (Lamb et 
345 al., 1999), but the factors behind the eyespot number variation observed in Leontynka spp. are

346 unknown. The reddish colour of the Leontynka eyespots suggests the presence of carotenoids

347 (similar to the eyespot of $C$. reinhardtii; Böhm \& Kreimer, 2020). In addition, searches of the $L$.

348 pallida transcriptome assembly revealed the presence of a homolog of the C. reinhardtii eyespot-

349 associated photosensor channelrhodopsin 1 (ChR1) that is the requires a carotenoid derivative,

350 retinal, as a chromophore (Petroutsos, 2017; Additional file 3: Table S1). The preservation of the

351 plastid-localized carotenoid biosynthetic pathway in non-photosynthetic eyespot-bearing

352 chlamydomonadaleans, namely certain Polytomella species and the strain $\mathrm{NrCl} 902$, has been noted

353 before (Asmail \& Smith, 2016; Kayama et al., 2020), and the same holds true for L. pallida based

354 on our analysis of its transcriptome assembly (Additional file 3: Table S1). Notably, like the

355 Polytomella species and the strain NrC1902 (Kayama et al., 2020), L. pallida has also retained

356 enzymes for the synthesis of plastoquinone, which serves as an electron acceptor in two reactions

357 of carotenoid biosynthesis, and the plastid terminal oxidase (PTOX), which recycles plastoquinone

358 (from its reduced form plastoquinole) by passing the electrons further to molecular oxygen

359 (Additional file 3: Table S1). Leontynka thus represents an independent case supporting the notion

360 that retention of the eyespot constraints the reductive evolution of a non-photosynthetic plastid.

361 Leontynka is significant not only as a novel non-photosynthetic group per se, but also as

362 an independent lineage within Chlamydomonadales lacking any close photosynthetic relatives.

363 Specifically, based on the phylogenetic analysis of plastome-encoded proteins, Leontynka

364 branches off between two large assemblages, each comprised of several major

365 chlamydomonadalean clades defined by Nakada et al. (2008). One of these assemblages

366 (potentially sister to Leontynka) is comprised of the Caudivolvoxa and Xenovolvoxa clades, the

367 other includes Reinhardtinia, Oogamochlamydinia, and the genus Desmotetra (Fig. 1a). The 
radiation of the Reinhardtinia clade itself was dated to 300 MYA (Herron et al., 2009), so the last common ancestor of Leontynka and any of its presently known closest photosynthetic relative must have existed even earlier. In other words, it is possible that Leontynka has been living without photosynthesis for hundreds of millions of years. The loss of photosynthesis in the four other

372 known colourless chlamydomonadalean lineages certainly does not trace that far in the past.

373 Specifically, the origin of Polytomella must postdate the radiation of Reinhardtinia, owing to the 374 position of the genus with this clade, whereas Polytoma sensu stricto ( $P$. uvella and relatives) has 375 close photosynthetic relatives (Chlamydomonas leiostraca, C. applanata etc.) within the clade 376 Polytominia in Caudivolvoxa (Fig. 1, Additional file 1: Fig. S1). Polytoma oviforme is specifically 377 related to the photosynthetic Chlamydomonas chlamydogama, together constituting a clade in 378 Xenovolvoxa that has not been formally recognised before and which we here designate 379 "Oviforminia" (Additional file 1: Fig. S1). Finally, the recently reported strain NrC1902 is closely 380 related to the photosynthetic Chlamydomonas pseudoplanoconvexa (Fig. 1A; Additional file 1: 381 Fig. S1). The independent phylogenetic position of L. pallida based on plastome-encoded proteins 382 is unlikely an artefact stemming from increased substitution rate of L. pallida plastid genes 383 manifested by the markedly longer branch of L. pallida in the tree compared to most other species 384 included in the analysis. Indeed, the branches of $P$. uvella and the strain $\mathrm{NrCl} 902$ are even longer 385 (Fig. 1B), yet both organisms are placed at positions consistent with the 18S rRNA gene tree (Fig. 386 1A; Additional file 1: Fig. S1). Nevertheless, whether Leontynka represents a truly ancient non387 photosynthetic lineage or whether it diverged from a photosynthetic ancestor rather recently needs 388 to be tested by further sampling of the chlamydomonadalean diversity, as we cannot rule out the 389 possibility that photosynthetic organisms closely related to the genus Leontynka are eventually 390 discovered. 
The presented considerations about the different ages of the separately evolved non-

392 photosynthetic chlamydomonadalean lineages are somewhat at odds with features of their

393 plastomes. Despite the presumably more recent loss of photosynthesis compared to Leontynka,

394 both $P$. uvella and the strain $\mathrm{NrCl} 902$ exhibit a more reduced set of plastid genes (Table 1), whereas

395 in Polytomella, plastome reduction triggered by photosynthesis loss has reached its possible

396 maximum, i.e., a complete disappearance of the genome. It is likely that factors other than

397 evolutionary time are contributing to the different degrees of plastome reduction in different

398 evolutionary lineages, although little is known in this regard. Compared to P. uvella and the strain

$399 \mathrm{NrC1902,} \mathrm{L.} \mathrm{pallida} \mathrm{has} \mathrm{preserved} \mathrm{one} \mathrm{gene} \mathrm{for} \mathrm{a} \mathrm{plastidial} \mathrm{ribosomal} \mathrm{protein} \mathrm{(rps2)} \mathrm{and,}$

400 intriguingly, all standard plastidial genes for ATP synthase subunits, complemented by three more

401 subunits encoded by the nuclear genome to allow for the assembly of a complete and presumably

402 functional complex. It was proposed that the retention of ATP synthase in certain non-

403 photosynthetic plastids is functionally linked to the retention of the twin-arginine protein

404 translocase (Tat; Kamikawa et al., 2015). The function of the translocase depends on a

405 transmembrane proton gradient, which in photosynthetic plastids is primarily generated by the

406 photosynthetic electron transport chain, whereas in non-photosynthetic ones its build-up would

407 depend solely on the function of ATP synthase working in the opposite direction, i.e. pumping

408 protons against the gradient at the expense of ATP. Interestingly, we found homologs of all three

409 Tat subunits (TatA, TatB, TatC) in the nuclear transcriptome of L. pallida (Additional file 3: Table

410 S1), providing further support to the hypothesis by Kamikawa et al. (2015). However, it must be

411 noted that certain members of the non-photosynthetic trebouxiophyte genus Prototheca possess

412 the plastidial ATP synthase in the absence of the Tat translocase (Suzuki et al., 2018), suggesting

413 that ATP synthase may be retained by a non-photosynthetic plastid for roles other than just 
414 supporting the function of the Tat system. Directly relevant for the retention of ATP synthase in

415 L. pallida might be its role in the functioning of the eyespot hypothesized in C. reinhardtii

416 (Schmidt et al., 2007).

417 The Tat translocase and the ATP synthase are both normally localised to the thylakoid 418 membrane. While thylakoids may seem dispensable in a non-photosynthetic plastid, it seems there 419 are putative thylakoids present in Leontynka, associated with the eyespot (Fig. $2 \mathrm{j}$ ). Indeed, in the 420 well-studied cases of $C$. reinhardtii and some other chlamydomonadalean algae, the layers of 421 pigment granules are organized on the surface of thylakoids closely apposed to the plastid envelope 422 (Kreimer, 1994; Böhm \& Kreimer, 2020). Interestingly, our searches of the L. pallida 423 transcriptome assembly revealed the presence of homologs of additional proteins functionally 424 associated with thylakoids. These include components of several additional thylakoid-associated 425 protein targeting or translocation systems (Schünemann et al., 2007; Skalitzky et al., 2011; Ziehe 426 et al., 2017), namely the plastidial SRP pathway (cpSRP54 and cpFtsY), ALB3 protein insertase, 427 and thylakoid-specific Sec translocase (Additional file 3: Table S1). Furthermore, we also found 428 in L. pallida homologs of proteins implicated in thylakoid biogenesis, such as VIPP1, FZL, THF1, 429 or SCO2 (Mechela et al., 2019; Additional file 3: Table S1). Interestingly, some of the 430 corresponding transcripts have very low read coverage or are even represented by incomplete 431 sequences, suggesting a low level of gene expression and presumably low abundance of the 432 respective proteins. These observations support the notion that the thylakoid system is preserved 433 in Leontynka plastids, however inconspicuous and likely reduced. Nevertheless, we cannot rule 434 out that at least some of these proteins or complexes may have relocalised to the inner bounding 435 membrane of the Leontynka plastid, or even to a cellular compartment other than the plastid (as 436 suggested for some of these proteins by the results of in silico targeting prediction (Additional file 

pathway, ALB3 insertase, and Tat and Sec translocases, and indeed, the physiological functions of the L. pallida leucoplast as a whole remain subjects for future research.

441 genomes. Generally, organellar genomes show a remarkable variation in the gene content, 442 architecture, and nucleotide composition, with most of them being AT-rich. The L. pallida 443 plastome is no exception in this respect, since its GC content is only $\sim 37 \%$. As noted by Smith 444 (2018), $98 \%$ of plastomes are under $200 \mathrm{kbp}$ and harbour modest amounts (<50\%) of non-coding 445 DNA. The L. pallida plastome, reaching $362.3 \mathrm{kbp}$, may not seem that impressive in comparison 446 with the giant plastomes recently reported from some photosynthetic species, including a distantly 447 related chlamydomonadalean Haematococcus lacustris (1.35 Mbp; Bauman et al., 2018) or certain 448 red algae (up to $1.13 \mathrm{Mbp}$; Muñoz-Gómez et al., 2017). However, it by far dwarfs plastomes of 449 all non-photosynthetic eukaryotes studied to date. The previous record holder, the plastome of 450 Polytoma uvella with $230 \mathrm{kbp}$ (Figueroa-Martinez et al., 2017), accounts for only two thirds of 451 the size of the L. pallida plastome. The difference is not only because of a higher number of genes 452 in the latter, but primarily because of a more extreme expansion of intergenic regions in L. pallida 453 (4.7 kbp on average) than in P. uvella $(3.0 \mathrm{kbp}$ on average; Table 1$)$. The plastome of the strain $454 \mathrm{NrCl} 902$ with its size of $176.4 \mathrm{kbp}$, while exhibiting the same gene content as the $P$. uvella 455 plastome, is much less extreme (Kayama et al., 2020), although still with the intergenic regions 456 substantially expanded as compared to the plastomes of non-photosynthetic trebouxiophytes 457 (Table 1).

Thus, despite its uniqueness, the organisation of the L. pallida plastome fits into the general 459 pattern observed in chlamydomonadalean algae, where plastomes in different lineages tend to 
460 increase in size by accumulating repetitive sequences (Gaouda et al., 2018; Smith, 2018). It was

461 suggested that the repeats are prone to double-strand breaks, which are then repaired by an error-

462 prone mechanism favouring repeat expansion (Smith, 2020a). However, the plastome of L. pallida

463 is bloated not only due to extreme proliferation of repetitive DNA in intergenic regions, but also

464 due to the expansion of some of them into the intronic regions and, much more surprisingly, even

465 into exons (Fig. 4). The biased orientation and phase of the insertions with respect to the coding

466 sequence and the reading frame avoid introduction of termination codons as well as rare codons

467 or codons for rare amino acids (C, W) into the coding sequences (Fig. 4 c, d, Additional file 2:

468 Note S4), which suggests that purifying selection eliminates those insertions that would disrupt or

469 reduce the efficiency of translation of the respective mRNAs. Still, exons provide an important

470 niche for the repeats: for example, for the "variant 8 " repeat, the exonic copies constitute $\sim 12.2 \%$

471 of the whole repeat population (compared to protein-coding sequences constituting $\sim 17.2 \%$ of the

472 total plastome length)! Such a massive proliferation of repeats to coding regions is unprecedented

473 to our knowledge, although a much less extensive invasion of a different repeat into coding

474 sequences was recently noticed in the plastome of another chlamydomonadalean alga,

475 Chlorosarcinopsis eremi (Smith 2020a). Here the repeats are found in small numbers in the genes

$476 f t s H, \operatorname{rpoC2}$, and $y c f 1$, paralleling the situation in L. pallida and consistent with the notion that

477 genes encoding proteins rich in poorly conserved regions are most likely to tolerate the invasion

478 of the repeats.

479 Recent sequencing of the mitogenome of $H$. lacustris, which is inflated by the

480 accumulation of repeats highly similar to those found in the plastome of the same species (Zhang

481 et al., 2019), provided the first evidence that error-prone repair of double-strand breaks leading to

482 repeat proliferation may operate also in chlamydomonadalean mitochondria. Smith (2020b) 
483 recently reported the presence of highly similar repeats in the mitogenome of another

484 chlamydomonadalean alga, Stephanosphaera pluvialis, and proposed horizontal gene transfer

485 between the $H$. lacustris and $S$. pluvialis lineages as a possible explanation for the sharing of

486 similar mitochondrial repeats by the two organisms. Our characterisation of the L. pallida

487 mitogenome, which is also repeat-rich and larger than any chlamydomonadalean algal

488 mitogenome sequenced to date except that from $H$. lacustris, revealed that mitogenome inflation

489 may be more common in Chlamydomonadales. However, in contrast to H. lacustris, the GC

490 content as well as the repeats in the two organellar genomes of L. pallida differ significantly

491 (Additional file 3: Table S2), so the evolutionary path leading to the parallel inflation of both

492 genomes in this lineage may have been completely different from the one manifested in $H$.

493 lacustris. Strikingly, the specific nature of the mitochondrial repeats in L. pallida entails the high

494 abundance of PQS in the mitogenome. G-quadruplexes are increasingly recognised as regulatory

495 structures (Hänsel-Hertsch et al., 2016), and they can form also in the mitogenomes, although their

496 role in mtDNA still needs to be elucidated (Falabella et al., 2019). However, the PQS abundance

497 in the L. pallida mitogenome is truly extreme and comparable only with the situation in the

498 mitogenome of the lycophyte S. moellendorffii (Additional file 3: Table S2). Both species are thus

499 interesting candidates for studying the role of G-quadruplexes in mitochondrial DNA.

501 Conclusions and future directions

502 Our study indicates that continued sampling of microbial eukaryotes is critical for further progress

503 in our knowledge of the phylogenetic diversity of life and for better understanding of the general

504 principles governing the evolution of organellar genomes. The specific factors contributing to the

505 propensity of chlamydomonadalean organellar genomes to accumulate repetitive sequences, 
reaching one of its extremes in L. pallida, remain unknown and may not be easy to define.

507 However, future research on Leontynka, including characterisation of organellar genomes of $L$.

508 elongata, may bring additional insights into the molecular mechanisms and evolutionary forces

509 shaping the organellar genomes in this group. It will also be important to perform a detailed

510 comparative analysis of the molecular machinery responsible for genome replication and

511 maintenance in Chlamydomonadales and other green algae. The transcriptome assembly reported

512 here for L. pallida will be instrumental not only in this enterprise, but will also serve as a resource

513 for exploring the full range of physiological roles of the plastid in the Leontynka lineage and may

514 help to further clarify the phylogenetic position of Leontynka within Chlamydomonadales. We

515 posit that Leontynka may become an important model system for analysing the evolutionary and

516 functional aspects of photosynthesis loss in eukaryotes with primary plastids.

518 Methods

519 Isolation, cultivation, and basic characterisation of new protist strains

520 Two strains, AMAZONIE and MBURUCU, were obtained from freshwater hypoxic sediment 521 samples collected in Peru and Argentina, respectively. The strains were cultivated and 522 morphologically characterised by light and transmission electron microscopy, using routine 523 methods. Basic molecular characterisation was achieved by determining partial sequences of the 524 rDNA operon. Further details are provided in Additional file 2: Methods S1-S3.

\section{Organellar genome and nuclear transcriptome sequencing}

527 Bacterial contamination in the AMAZONIE culture was minimised by filtration, and DNA and 528 RNA were extracted using standard protocols detailed in Additional file 2: Methods S4. Nanopore 
sequencing was performed using $4 \mu \mathrm{g}$ of genomic DNA. The DNA was sheared at $20 \mathrm{kbp}$ using

530 Covaris g-TUBE (Covaris) according to the manufacturer's protocol. After shearing, two libraries

531 were prepared using Ligation Sequencing Kit from Oxford Nanopore Technologies (SQK-

532 LSK108). The prepared library was loaded onto a R9.4.1 Spot-On Flow cell (FLO-MIN106).

533 Sequencing was performed on a MinION Mk1B machine for 48 hours using the MinKNOW 2.0

534 software. Basecalling was performed using Guppy 3.0.3 with the Flip-flop algorithm. Illumina

535 sequencing of the genomic DNA was performed using $1 \mu \mathrm{g}$ of genomic DNA with the Illumina

536 HiSeq 2000 (2x150bp) paired-end technology with libraries prepared using TruSeq DNA PCR-

537 Free (Illumina, San Diego, CA) at Macrogen Inc. (Seoul, South Korea). The transcriptome was

538 sequenced using the HiSeq 2000 (2x100bp) paired-end technology with libraries prepared using

539 the TruSeq RNA sample prep kit v2 (Illumina, San Diego, CA) at Macrogen Inc. (Seoul, South

540 Korea).

542 Organellar genome and nuclear transcriptome assembly

543 Raw Illumina sequencing reads were trimmed with Trimmomatic v0.32 (Bolger et al., 2014).

544 Initial assembly of the Oxford Nanopore data was performed using Canu v1.7 with the

545 corMaxEvidenceErate set to 0.15 (Koren et al., 2017). After assembly, the plastome-derived

546 contigs were identified using BLAST (Altschul et al., 1997) with the Chlamydomonas reinhardtii

547 plastome as a query. Nine putative plastid genome sequences were selected and polished using the

548 raw nanopore reads with Nanopolish (Loman et al., 2015) followed by polishing with Illumina

549 reads with Pilon v1.22 (Walker et al., 2014). After polishing of the contigs, the Illumina reads

550 were re-mapped onto them, and the mapped reads were extracted and used as an input in Unicycler

551 v0.4.8 (Wick et al., 2017) together with the nanopore reads. Unicycler generated a single circular 
552 contig of 362,307 bp. For the mitogenome, a single linear contig was identified in the Canu

553 assembly with BLAST with standard mitochondrial genes as queries; the contig sequence was

554 polished using the same method as described above, but it remained linear after a subsequent

555 Unicycler run. However, direct inspection of the contig revealed highly similar regions (about

$5565,600 \mathrm{bp}$ in length) at both termini. The terminal regions were further polished by mapping of

557 Illumina genomic reads using BWA (Li, 2013) and SAMtools (Li et al., 2009) followed by manual

558 inspection in Tablet (Milne et al., 2016), which increased the sequence similarity of the termini to

$55997.7 \%$ (along the region of $5,771 \mathrm{bp}$ ).

560 Illumina genomic reads were also assembled separately with the SPAdes Genome

561 assembler v3.10.1 (Bankevich et al., 2012) and used for cleaning the transcriptomic data as

562 follows. Contaminant bacterial contigs $>400,000$ bp that were identified with BLAST in the

563 SPAdes (16 contigs) and Canu assemblies (11 contigs), together with published genome

564 assemblies of close relatives of bacteria identified in the AMAZONIE culture (Curvibacter

565 lanceolatus ATCC 14669, Bacteroides luti strain DSM 26991, and Paludibacter jiangxiensis

566 strain NM7), were used for RNA-seq read mapping (Hisat2 2.1.0; Kim et al., 2015) to identify and

567 remove bacterial transcriptomic reads that survived the filtration of the culture and polyA

568 selection. This procedure removed $\sim 4 \%$ of the reads. Cleaned reads were used for transcriptome

569 assembly with the rnaSPAdes v3.13.0 using k-mer size of 55bp (Bushmanova et al., 2019).

\section{Annotation of organellar genomes and other sequence analyses}

572 Initial annotation of both the plastid and mitochondrial genomes of the strain AMAZONIE were

573 obtained using MFannot (http://megasun.bch.umontreal.ca/cgi-bin/dev_mfa/mfannotInterface.pl).

574 The program output was carefully checked manually, primarily by relying on BLAST searches, to 
575 find possible missed genes, to validate or correct the assessment of the initiation codons, to fix the

576 delimitation of introns, and to ensure that all genes were properly named. ORFs lacking discernible

577 homologs (as assessed by HHpred; Zimmermann et al., 2018) encoding proteins shorter than 150

578 amino acid residues and ORFs consisting mostly of sequence repeats were omitted from the

579 annotation. Distribution of repeats within the organellar genomes and comparison of repeats

580 between organellar genomes of L. pallida and other selected chlamydomonadaleans were analysed

581 using the dottup programme from the EMBOSS package (http://www.bioinformatics.nl/cgi-

582 bin/emboss/dottup). Detailed analyses of imperfect palindromes and G-quadruplexes were

583 performed using the Palindrome analyzer (Brázda et al., 2016) and the G4hunter web-based server

584 (Brázda et al., 2019). The Palindrome analyzer was used to search for motifs 8-100 bp in length

585 with spacers 0-10 bp, and a maximum of one mismatch in the palindrome. The G4hunter web-

586 based server was used under the default settings, i.e., window=25 and threshold=1.2.

587 To understand the position of amino acid stretches encoded by the characteristic repeats

588 that have invaded the coding sequence of the $\mathrm{fts} H$ gene, the tertiary structure of the encoded protein

589 was predicted by homology modelling using the Phyre2 program

590 (http://www.sbg.bio.ic.ac.uk/phyre2/html/page.cgi?id=index; Kelley et al., 2015). The secondary

591 structure of the ITS2 region was modelled manually according to the consensus secondary ITS2

592 structure of two green algae (Caisová et al., 2013), visualised by VARNA software (Darty et al.,

593 2009), and manually edited in a graphical editor. Homologs of nucleus-encoded plastidial proteins

594 of specific interest were searched in the L. pallida transcriptome assembly by using TBLASTN

595 and the respective proteins sequences from Arabidopsis thaliana or C. reinhardtii (selected based

596 on the information from the literature or keyword database searches). Significant hits (E-value

$597 \leq 1 \mathrm{e}-5$ ) were evaluated by BLASTX searches against the NCBI non-redundant protein sequence 
database to filter out bacterial contaminants and sequences corresponding to non-orthologous members of broader protein families. Subcellular localization (for complete sequences only) was assessed by using TargetP-2.0 (https://services.healthtech.dtu.dk/service.php?TargetP-2.0;

601 Almagro Armenteros et al., 2019) and PredAlgo (http://lobosphaera.ibpc.fr/cgi-

602 bin/predalgodb2.perl?page=main; Tadrif et al., 2012).

\section{Phylogenetic analyses}

605 Multiple sequence alignment of 18S rRNA gene relying on a total of 201 chlorophyte OTUs was computed using MAFFT v7 (Katoh et al., 2019) and trimmed manually. The $18 \mathrm{~S}$ rRNA sequence

607 from Polytoma oviforme available in GenBank (U22936.1) was proposed to be chimeric (Nakada 608 et al., 2008), but given the relevance of this organism for our analysis, we included it, masking the 609 regions putatively derived from a different source by strings of N. Maximum likelihood tree 610 inference was performed using IQ-TREE multicore v1.6.12 (Nguyen et al., 2015) under 611 TIM2+F+I+G4 model with 100 non-parametric bootstrap replicates. For multigene analysis, 612 alignments of conserved plastome-encoded proteins used previously (Fučíková et al., 2019) were 613 updated by adding the respective homologs from L. pallida and thirteen additional relevant 614 chlorophycean taxa not represented in the initial dataset. On the other hand, sequences representing 615 the OCC clade of Chlorophyceae (evidently only distantly related to L. pallida based on the 18S 616 rRNA gene phylogeny and morphological features) to keep the size of the dataset easier to analyse 617 with a complex substitution model. For the final matrix, a subset of 24 proteins (all having their $L$. 618 pallida representative) were used. Multiple alignments of the homologous amino acid sequences 619 were built using MAFFT v7.407 with the L-INS-i algorithm (Katoh \& Standley, 2013) and 620 manually trimmed to exclude unreliably aligned regions. The final concatenated matrix comprised 
6215,020 amino acid residues. The tree was built using PhyloBayes v4.1 (Lartillot et al., 2013) under

622 the CAT + GTR model of sequence evolution, with two independent chains that converged at

62315,298 generations with the largest discrepancy in posterior probabilities (PPs) (maxdiff) of

6240.0535238 (at burn-in of 20\%). The maximum likelihood (ML) tree was inferred with IQ-TREE

625 multicore v1.6.12 using the $\mathrm{LG}+\mathrm{C} 60+\mathrm{F}+\mathrm{G} 4$ substitution model. Statistical support was assessed

626 with 100 IQ-TREE non-parametric bootstraps with correction and PhyloBayes posterior 627 probabilities.

629 Supplementary information

630 Supplementary information accompanies this paper at XXXXXXX.

632 Additional file 1: Supplementary Figs S1-S9. Fig. S1. Maximum likelihood phylogenetic tree

633 (RAxML, GTRGAMMA+I substitution model) of $18 \mathrm{~S}$ rRNA gene sequences from

634 Chlorophyceae. Fig. S2. Predicted secondary structure of the ITS2 region of Leontynka pallida,

635 with differences in the corresponding region of Leontynka elongata mapped onto it. Fig. S3.

636 Leontynka pallida under the light microscope. Fig. S4. Leontynka elongata under the light

637 microscope. Fig. S5. Ultrastructure of Leontynka elongata (a-f) and Leontynka pallida (g-i). Fig.

638 S6. Occurrence of the "variant 8" repeat in the FtsH protein of Leontynka pallida mapped on its

639 predicted structure. Fig. S7. Alignment of the highly similar terminal regions of the originally

640 assembled linear mitogenome contig. Fig. S8. Occurrence of the "variant 8" repeat (translated in

641 reading frame +0 as KDKPANLTS and -0 as KEVSFAGLSL) in variable region of protein

642 sequence of the ribosomal protein Rps8 from Leontynka pallida (full protein alignment together

643 with representatives of other chlamydomonadalean algae). 
Additional file 2: Supplementary Notes S1-S5 and supplementary Methods S1-S4. Note S1.

646 Taxonomic descriptions. Note S2. Further details on the morphology and ultrastructure of

647 Leontynka spp. Note S3. Further details on various kinds of repeats in the plastome of L. pallida.

648 Note S4. Further details on the repeat insertions in L. pallida plastid coding sequences. Note S5.

649 Differential diagnosis of Leontynka spp. with regard to previously described colourless

650 chlamydomonadalean taxa. Methods S1. Isolation and cultivation of strains. Methods S2. Light

651 and transmission electron microscopy. Methods S3. Amplification and sequencing of 18S and ITS

652 rDNA regions. Methods S4. DNA and RNA isolation.

654 Additional file 3: Supplementary Tables S1-S7. Table S1. Nuclear transcripts from Leontynka

655 pallida specifically discussed in the paper. Table S2. Comparison of GC content, number of 656 imperfect palindromes, and potential quadruplex-forming sequences in selected organellar 657 genomes. Table S3. Strong codon usage bias in the mitochondrial genome of Leontynka pallida.

658 Table S4. Relative frequency of amino acids with GC-rich codons (G, A, R, P) in proteins encoded 659 by different mitogenomes. Table S5. Relative frequency of codons in plastid genes of Leontynka 660 pallida. Table S6. Relative frequency of amino acids in proteins encoded by the plastome of 661 Leontynka pallida. Table S7. The most abundant imperfect palindrome in the Leontynka pallida 662 plastome that is missing in exons.

\section{Declarations}

665 Ethics approval and consent to participate

666 Not applicable. 


\section{Consent for publication}

669 Not applicable.

670

671 Competing interests

672 The authors declare no competing interests.

673

\section{Availability of data and materials}

675 Sequences determined in this study are available from GenBank with the following accession

676 numbers: \#\#\#\#\# and \#\#\#\#\#- partial nuclear rDNA sequences (18S rRNA-ITS1-5.8S rRNA-ITS2)

677 from L. pallida and L. elongata, respectively; \#\#\#\# and \#\#\#\# - plastid and mitochondrial genome

678 sequence from L. pallida; \#\#\#\#\# - transcriptome assembly from L. pallida. The cultures of 679 Leontynka spp. investigated in this study are available upon request.

680

\section{$681 \quad$ Funding}

682 This work was supported by the Czech Science Foundation project 17-21409S (to M.E.) and the 683 project "CePaViP", supported by the European Regional Development Fund, within the 684 Operational programme for Research, Development and Education 685 (CZ.02.1.01/0.0/0.0/16_019/0000759). TP was also supported by the Charles University (UNCE 686 204069).

687

688 Authors' contribution 
689 TP, DB, IČ and ME conceived the original research plans; TP, SCT, KZ, EZ, and KJ obtained

690 nucleic acids for sequencing; SCT and TP obtained Oxford Nanopore data and generated

691 organellar genome assemblies; EZ and IC isolated the strains; TP and DB carried out the

692 morphological characterisation of the strains; DB and NY obtained the TEM data; MS assembled

693 the transcriptome; TP, DB, and TŠ carried out phylogenetic analyses; TP, IČ and ME analysed

694 and annotated the organellar genome sequences; IČ and ME supervised the work of junior

695 researchers and obtained funding; TP, DB and ME drafted the manuscript; all authors contributed

696 to the final version of the text; ME agreed to serve as the author responsible for contact and

697 ensuring communication.

699 Acknowledgements

700 We are thankful to Karolina Fučíková for providing alignments of chlorophycaean plastid proteins 701 and Petr Janšta for collecting the sample MBURUCU.

702

703 References

704 Almagro Armenteros JJ, Salvatore M, Emanuelsson O, Winther O, von Heijne G, Elofsson

705 A, Nielsen H. 2019. Detecting sequence signals in targeting peptides using deep learning.

$706 \quad$ Life Science Alliance 2: e201900429.

707 Altschul SF, Madden TL, Schäffer AA, Zhang J, Zhang Z, Miller W, Lipman DJ. 1997.

708 Gapped BLAST and PSI-BLAST: a new generation of protein database search programs.

$709 \quad$ Nucleic Acids Research 25: 3389-3402.

710 Asmail SR, Smith DR. 2016. Retention, erosion, and loss of the carotenoid biosynthetic pathway

711 in the nonphotosynthetic green algal genus Polytomella. New Phytologist 209: 899-903. 
Bankevich A, Nurk S, Antipov D, Gurevich AA, Dvorkin M, Kulikov AS, Lesin VM, algorithm and its applications to single-cell sequencing. Journal of Computational Biology 19: 455-477. generation sequencing of Haematococcus lacustris reveals an extremely large 1.35-

Böhm M, Kreimer G. 2020. Orient in the World with a Single Eye: The Green Algal Eyespot and Phototaxis. In: Cánovas FM, Lüttge U, Risueño MC, Pretzsch H (eds) Progress in Botany Vol. 82. Progress in Botany, vol 82. Springer, Cham.

Bolger AM, Lohse M, Usadel B. 2014. Trimmomatic: a flexible trimmer for Illumina sequence data. Bioinformatics 30: 2114-2120.

Boer PH, Gray MW. 1988. Scrambled ribosomal RNA gene pieces in Chlamydomonas reinhardtii mitochondrial DNA. Cell 55: 399-411. web application: a web server for G-quadruplex prediction. Bioinformatics 35: 3493-3495. analyser - A new web-based server for predicting and evaluating inverted repeats in nucleotide sequences. Biochemical and Biophysical Research Communications 478: 17391745. topology and structure Nucleic Acids Research 34: 5402-5415. 
734 Bushmanova E, Antipov D, Lapidus A, Prjibelski AD. 2019. rnaSPAdes: a de novo 735 transcriptome assembler and its application to RNA-Seq data. GigaScience 8: giz100.

736 Caisová L, Marin B, Melkonian M. 2013. A consensus secondary structure of ITS2 in the Chlorophyta identified by phylogenetic reconstruction. Protist 164: 482-496.

Coleman AW. 2000. The significance of a coincidence between evolutionary landmarks found in mating affinity and a DNA sequence. Protist 151: 1-9.

Darty K, Denise A, Ponty Y. 2009. VARNA: Interactive drawing and editing of the RNA secondary structure. Bioinformatics 25: 1974-1975.

Del Vasto M, Figueroa-Martinez F, Featherston J, González MA, Reyes-Prieto A, Durand PM, Smith DR. 2015. Massive and widespread organelle genomic expansion in the green algal genus Dunaliella. Genome Biology and Evolution 7: 656-663.

Denovan-Wright EM, Lee RW. 1994. Comparative structure and genomic organization of the discontinuous mitochondrial ribosomal RNA genes of Chlamydomonas eugametos and Chlamydomonas reinhardtii. Journal of Molecular Biology 241: 298-311. organization of ATP synthase in intact mitochondria determined by cryo-electron tomography. Biochimica et Biophysica Acta (BBA)-Bioenergetics 1797: 272-277.

Ettl H. 1983. Chlorophyta I: Phytomonadina. In: Ettl H, Gerloff J, Heynig H, Mollenhauer D (eds.), Süßwasserflora von Mitteleuropa. Band 9. Stuttgart: Gustav Fischer Verlag. 807 p.

Falabella M, Fernandez RJ, Johnson FB, Kaufman BA. 2019. Potential roles for Gquadruplexes in mitochondria. Current Medicinal Chemistry 26: 2918-2932. 
Figueroa-Martinez F, Nedelcu AM, Smith DR, Reyes-Prieto A. 2015. When the lights go out: the evolutionary fate of free-living colorless green algae. New Phytologist 206: 972-982.

Figueroa-Martinez F, Nedelcu AM, Smith DR, Reyes-Prieto A. 2017. The plastid genome of Polytoma uvella is the largest known among colorless algae and plants and reflects contrasting evolutionary paths to nonphotosynthetic lifestyles. Plant Physiology 173: 932-

Fučíková K, Lewis PO, Neupane S, Karol KG, Lewis LA. 2019. Order, please! Uncertainty in the ordinal-level classification of Chlorophyceae. PeerJ 7: e6899.

Gaffal KP, Schneider GJ. 1980. Numerical, morphological and topographical heterogeneity of the chondriome during the vegetative life cycle of Polytoma papillatum. Journal of Cell Science 46: 299-312. genome expansion in volvocine green algae. Genome Biology and Evolution 10: 2248-2254.

Greiner S, Lehwark P, Bock R. 2019. OrganellarGenomeDRAW (OGDRAW) version 1.3.1: expanded toolkit for the graphical visualization of organellar genomes. Nucleic Acids Research 47: W59-W64

Gruber HE, Rosario B. 1974. Variation in eyespot ultrastructure in Chlamydomonas reinhardi (ac-31). Journal of Cell Science 15: 481-494.

Hadariová L, Vesteg M, Hampl V, Krajčovič J. 2018. Reductive evolution of chloroplasts in non-photosynthetic plants, algae and protists. Current Genetics 64: 365-387. 
Hänsel-Hertsch R, Beraldi D, Lensing SV, Marsico G, Zyner K, Parry A, Di Antonio M, Pike chromatin. Nature Genetics 48: 1267-1272.

Hecht J, Grewe F, Knoop V. 2011. Extreme RNA editing in coding islands and abundant microsatellites in repeat sequences of Selaginella moellendorffii mitochondria: The root of frequent plant mtDNA recombination in early tracheophytes. Genome Biology and Evolution

Herron MD, Hackett JD, Aylward FO, Michod RE. 2009. Triassic origin and early radiation of multicellular volvocine algae. Proceedings of the National Academy of Sciences, USA 106: $3254-3258$.

Kamikawa R, Tanifuji G, Ishikawa SA, Ishii K, Matsuno Y, Onodera NT, Ishida K, Hashimoto T, Miyashita H, Mayama S et al. 2015. Proposal of a twin arginine translocator system-mediated constraint against loss of ATP synthase genes from nonphotosynthetic plastid genomes. [Corrected]. Molecular Biology and Evolution 32: 2598-2604. reductive evolution of plastid electron transport systems. BMC Biology 18: 126. protein modeling, prediction and analysis. Nature Protocols 10: 845-858. 
Kim D, Langmead B, Salzberg S. 2015. HISAT: a fast spliced aligner with low memory requirements. Nature Methods 12: 357-360.

Koren S, Walenz BP, Berlin K, Miller JR, Bergman NH, Phillippy AM. 2017. Canu: scalable and accurate long-read assembly via adaptive k-mer weighting and repeat separation. Genome Research 27: 722-736.

Kreimer G. 1994. Cell biology of phototaxis in flagellate algae. International Review of Cytology 148: $229-310$.

Lamb MR, Dutcher SK, Worley CK, Dieckmann CL. 1999. Eyespot-assembly mutants in Chlamydomonas reinhardtii. Genetics 153: 721-729.

Lang NJ. 1963. Electron microscopic demonstration of plastids in Polytoma. Journal of Protozoology 10: 333-339.

Lavrov DV, Adamski, M, Chevaldonne, P, Adamska, M. 2016. Extensive mitochondrial mRNA editing and unusual mitochondrial genome organization in calcaronean sponges. Current Biology 26: 86-92.

Lartillot N, Rodrigue N, Stubbs D, Richer J. 2013. PhyloBayes MPI: phylogenetic reconstruction with infinite mixtures of profiles in a parallel environment. Systematic

Li H, Handsaker B, Wysoker A, Fennell T, Ruan J, Homer N, Marth G, Abecasis G, Durbin R, 1000 Genome Project Data Processing Subgroup. 2009. The sequence alignment/map format and SAMtools. Bioinformatics 25: 2078-2079.

821 Li H. 2013. Aligning sequence reads, clone sequences and assembly contigs with BWA-MEM. arXiv preprint arXiv:1303.3997. 
823 Loman NJ, Quick J, Simpson JT. 2015. A complete bacterial genome assembled de novo using only nanopore sequencing data. Nature Methods 12: 733-735.

825 Mechela A, Schwenkert S, Soll J. 2019. A brief history of thylakoid biogenesis. Open Biology 9 : 180237.

Milne I, Bayer M, Stephen G, Cardle L, Marshall D. 2016. Tablet: visualizing next-generation sequence assemblies and mappings. Methods in Molecular Biology 1374: 253-268. largest and most divergent plastid genomes known. Current Biology 27: 1677-1684.e4.

Nakada T, Misawa K, Nozaki H. 2008. Molecular systematics of Volvocales (Chlorophyceae, Chlorophyta) based on exhaustive $18 \mathrm{~S}$ rRNA phylogenetic analyses. Molecular Phylogenetics and Evolution 48: 281-291.

Nguyen LT, Schmidt HA, von Haeseler A, Minh BQ. 2015. IQ-TREE: a fast and effective stochastic algorithm for estimating maximum-likelihood phylogenies. Molecular Biology and Evolution 32: 268-274.

Petroutsos D. 2017. Chlamydomonas Photoreceptors: Cellular Functions and Impact on Physiology. In: Hippler M. (eds) Chlamydomonas: Biotechnology and Biomedicine. Microbiology Monographs, vol 31. Springer, Cham.

Schmidt M, Luff M, Mollwo A, Kaminski M, Mittag M, Kreimer G. 2007. Evidence for a specialized localization of the chloroplast ATP-synthase subunits $\alpha, \beta$, and $\gamma$ in the eyespot apparatus of Chlamydomonas reinhardtii (Chlorophyceae). Journal of Phycology 43: 284294. 
845 Schünemann D. 2007. Mechanisms of protein import into thylakoids of chloroplasts. Biological Chemistry 388: 907-915.

Sibbald SJ, Archibald JM. 2020. Genomic insights into plastid evolution. Genome Biology and Evolution 12: 978-990.

Siu C, Swift H, Chiang K. 1976. Characterization of cytoplasmic and nuclear genomes in the colorless alga Polytoma. Journal of Cell Biology 69: 352-370.

Skalitzky CA, Martin JR, Harwood JH, Beirne JJ, Adamczyk BJ, Heck GR, Cline K, Fernandez DE. 2011. Plastids contain a second sec translocase system with essential functions. Plant Physiology 155: 354-369.

Smith, DR. 2012. Updating our view of organelle genome nucleotide landscape. Frontiers in Genetics 3: 175.

Smith DR, Hua J, Lee RW. 2010. Evolution of linear mitochondrial DNA in three known lineages of Polytomella. Current Genetics 56: 427-438.

858 Smith DR, Hua J, Archibald JM, Lee RW. 2013. Palindromic genes in the linear mitochondrial genome of the nonphotosynthetic green alga Polytomella magna. Genome Biology and Evolution 5: 1661-1667.

Smith DR, Lee RW. 2014. A plastid without a genome: evidence from the nonphotosynthetic green algal genus Polytomella. Plant Physiology 164: 1812-1819.

Smith DR. 2018. Haematococcus lacustris: the makings of a giant-sized chloroplast genome. AoB Plants 10: ply058.

Smith DR. 2020a. Can green algal plastid genome size be explained by DNA repair mechanisms? Genome Biology and Evolution 12: 3797-3802. 
867 Smith DR. 2020b. Common repeat elements in the mitochondrial and plastid genomes of green algae. Frontiers in Genetics 11: 465.

Smith DR, Craig, R. J. 2021. Does mitochondrial DNA replication in Chlamydomonas require a reverse transcriptase? New Phytologist 229: 1192-1195.

Suzuki S, Endoh R, Manabe RI, Ohkuma M, Hirakawa Y. 2018. Multiple losses of Prototheca. Scientific Reports 8: 940. prediction tool dedicated to green algae. Molecular Biology and Evolution 29: 3625-3639.

Turmel M, Lemieux C. 2018. Chapter six - Evolution of the plastid genome in green algae. Advances in Botanical Research 85: 157-193. Wortman J, Young SK et al. 2014. Pilon: an integrated tool for comprehensive microbial variant detection and genome assembly improvement. PLoS One 9: e112963.

Webster DA, Hackett DP, Park RB. 1968. The respiratory chain of colorless algae: III. Electron microscopy. Journal of Ultrastructural Research 21: 514-523.

884 Wick RR, Judd LM, Gorrie CL, Holt KE. 2017. Unicycler: resolving bacterial genome assemblies from short and long sequencing reads. PLoS Computational Biology 13: $\mathrm{e} 1005595$.

Wolf M, Chen S, Song J, Ankenbrand M, Müller T. 2013. Compensatory base changes in ITS2 secondary structures correlate with the biological species concept despite intragenomic variability in ITS2 sequences--a proof of concept. PLoS One 8: e66726. 

made up of nearly identical repetitive sequences. Current Biology 29: R736-R737. chloroplast SRP system. Biological Chemistry 398: 653-661. new HHpred server at its core. Journal of Molecular Biology 430: 2237-2243. 
911 Table 1. Basic characteristics of plastomes of Leontynka pallida, selected other non-

912 photosynthetic chlorophytes and the photosynthetic Chlamydomonas reinhardtii and

913 Haematococcus lacustris for comparison.

914

915

\begin{tabular}{|c|c|c|c|c|c|c|c|c|c|}
\hline \multirow[b]{2}{*}{ species } & \multicolumn{2}{|c|}{ plastomes } & \multicolumn{2}{|c|}{ genes } & \multicolumn{2}{|c|}{ coding DNA** } & \multicolumn{3}{|c|}{ intergenic regions } \\
\hline & $\begin{array}{l}\text { accession } \\
\text { number }\end{array}$ & size (bp) & $\begin{array}{l}\text { protein- } \\
\text { coding* }\end{array}$ & tRNAs & GC (\%) & $\begin{array}{c}\text { total } \\
\text { length } \\
(\%)\end{array}$ & $\begin{array}{l}\text { GC } \\
(\%)\end{array}$ & $\begin{array}{c}\text { average } \\
\text { length } \\
\text { (bp) }\end{array}$ & $\begin{array}{c}\text { total } \\
\text { length } \\
(\%)\end{array}$ \\
\hline Leontynka pallida & \#\#\#\#\# & 362,307 & 32 & 26 & 35 & 18 & 37 & 4,726 & 80 \\
\hline Polytoma uvella & KX828177.1 & 230,207 & 25 & 27 & 40 & 20 & 19 & 2,998 & 68 \\
\hline $\begin{array}{l}\text { Volvocales sp. } \\
\mathrm{NrCl} 902\end{array}$ & LC516060.1 & 176,432 & 25 & 30 & 36.1 & 32.6 & 42.8 & 1,944 & 60.9 \\
\hline Helicosporidium sp. & DQ398104.1 & 37,454 & 26 & 25 & 27.6 & 95.4 & 13.4 & 33.8 & 4.4 \\
\hline $\begin{array}{l}\text { Chlamydomonas } \\
\text { reinhardtii }\end{array}$ & NC_005353.1 & 203,828 & 69 & 29 & 34.6 & 43 & 34 & 923 & 48.5 \\
\hline $\begin{array}{l}\text { Haematococcus } \\
\text { lacustris }(\dagger)\end{array}$ & NC_037007.1 & $1,352,306$ & 63 & 28 & 38.6 & 6.8 & 51 & 12,326 & 91.15 \\
\hline
\end{tabular}

$916 *$ does not include ORFs inside introns

$917 * *$ does not include introns and ORFs inside introns, but includes tRNAs and rRNAs

918 (†) since the annotation of the H. lacustris plastome available in the respective GenBank record is highly incomplete, the values presented are based on a reannotation obtained by using MFannot (with a genetic code translating UGA as Trp, based on the insights by Smith, 2018). 


\section{$928 \quad$ Figure legends}

929 Fig. 1 Phylogenetic position of the new genus of non-photosynthetic green algae, Leontynka. Non-

930 photosynthetic taxa in Chlamydomonadales are highlighted in violet. (a) Maximum likelihood

931 phylogenetic tree (IQ-TREE, TIM2+F+I+G4 substitution model) of 18S rRNA gene sequences

932 from Chlamydomonadales and related chlorophytes. Non-parametric bootstrap support values

933 calculated from 100 replicates are shown when $\geq 50$. Previously demarcated main clades (Nakada

934 et al., 2008) are collapsed and the outgroup taxa are not shown for simplicity. The full version of

935 the tree is provided as Additional file 1: Fig. S1. (b) Phylogenetic analysis of a concatenated dataset

936 of 24 conserved plastome-encoded proteins (5,020 amino acid positions) from

937 Chlamydomonadales, including Leontynka pallida, and the sister order Sphaeropleales (sensu lato;

938 see Fučíková et al., 2019). The tree topology was inferred using PhyloBayes (CAT+GTR

939 substitution model), branch support values correspond to posterior probability (from PhyloBayes)

940 / maximum likelihood bootstrap analysis (IQ-TREE, LG+C60+F+G4 substitution model, 100 non-

941 parametric bootstrap replicates). Black dots represent full support obtained with both methods,

942 asterisks denote bootstrap support values $<50$.

944 Fig. 2 Light and transmission electron microscopy of Leontynka pallida (a-e) and Leontynka

945 elongata $(\mathrm{f}-\mathrm{j})$. Note the difference in the cell shape between the two species and the presence of a

946 single $(a, f, h)$ or two (b, d, g, i, j) eyespots. Lipid droplets were also detected within the cells (d,

947 h, j). Abbreviations: bb - basal body; cv - contractile vacuole; e - eyespot; L - lipid droplet; m -

948 mitochondrion; $\mathrm{N}$ - nucleus; $\mathrm{n}$ - nucleolus; $\mathrm{s}$ - starch. Arrows point to thylakoids; asterisks mark

949 plastid "bridges" connecting separate compartments; arrowheads show membranous inclusions.

$950 \quad$ Scale bars: $a-c, f-g=10 \mu \mathrm{m} ; \mathrm{d}, \mathrm{i}=0.2 \mu \mathrm{m} ; \mathrm{e}=0.5 \mu \mathrm{m} ; \mathrm{j}=1 \mu \mathrm{m}$. 
952 Fig. 3 Characteristics of the organellar genomes of Leontynka pallida. (a) Gene map of the $L$.

953 pallida plastid genome. Genes are shown as squares (coloured according to the functional

954 category; see the graphical legend in the left bottom corner) on the inner or outer side of the outer

955 circle depending on their orientation (transcription in the clockwise or counter-clockwise direction,

956 respectively; see the grey arrows). Genes marked with an asterisk contain introns. The inner circle

957 represents a GC content plot. (b) Sequence self-similarity plot of the L. pallida plastome (ptDNA).

958 (c) Map of the L. pallida mitochondrial genome. The display convention is the same as for the

959 plastid genome. (d) Sequence self-similarity plot of the L. pallida mitochondrial genome

960 (mtDNA). (e) L. pallida plastome-mitogenome similarity plot. All similarity plots were generated

961 by using the word size of 15 bp and black dots represent the occurrence of the same word at the

962 places compared. The organellar genome maps (a, c) were visualised by using OGDRAW v1.3.1

963 (Greiner et al., 2019).

964

965 Fig. 4 Distribution of repeats in organellar genomes of Leontynka pallida. (a) Most abundant

966 imperfect palindromes and their characteristics. The "Spacer" corresponds to the presumed loop

967 separating the palindromic regions presumably pairing to form a stem structure. The "Mismatch"

968 column indicates the number of positions that deviate from a perfect palindrome. The occurrence

969 of the repeats is given for the plastome (ptDNA) and mitogenome (mtDNA), with the number of

970 cases indicated for the whole organellar genome and separately for exons in protein-coding genes.

971 In two cases of mitogenome repeats, two variants - a shorter and a longer - are considered, with

972 the latter indicated in parentheses. (b) Distribution of the imperfect palindrome

973 AAGCCAGC|NNN|GCTGACTT and its most common variants within exons of the plastome. The 
974 numbers show the abundance of the given repeat in direct / reverse complement orientation

975 (relative to the coding sequence). In the case of "variant 1", the repeat has the same sequence in

976 both directions, so only one number per gene is presented. Note that the variants considered are

977 not mutually exclusive alternatives but correspond to nested categories with a different degree or

978 relaxation of the sequence pattern. (c) Characterisation of repeats from (b) and their abundance in

979 various regions of the plastome and the mitogenome of L. pallida as well as other plastomes of

980 Chlamydomonadales deposited in NCBI databases. The numbers show the abundance of the given

981 repeat in direct / reverse complement orientation (relative to the coding sequence in the case of

982 exons, or relative to the DNA strand corresponding to the reference organellar sequence in case of

983 the values for the whole organellar genome). (d) Occurrence of the "variant 8" repeat (translated

984 in the reading frame +0 as KDKPANLTS) in a variable region of the ribosomal protein Rps8

985 (detail; the full alignment is available as Additional file 1: Fig. S8). (e) Occurrence of the "variant

986 4" repeat in protein-coding sequences and its translation in all six reading frames. The category of

987 rare codons ("rare 2\%") is defined as the sum of the least used codons together representing less

988 than $2 \%$ of all codons in the plastome $(100 \%=19,899$ codons $)$; the categories of the $4 \%, 10 \%$,

989 and $20 \%$ rarest codons and more than $50 \%$ of most frequent codons are defined similarly (listed

990 in Additional file 3: Table S5). The numbers indicated for the "codon usage" correspond to the

991 minimal number of the codons of the respective category present in the respective reading frame,

992 with the "max X" numbers indicating the maximal number of such codons depending on the actual

993 nucleotide sequence of the degenerated "variant 4" repeat. Note that is some same the theoretical

994 maximal number is not observed in the actual L. pallida plastid gene sequences (see the asterisks).

995 The column "Rare AA" indicates the occurrence of amino acids belonging to the category of amino

996 acids generally rarely used in plastome-encoded proteins in L. pallida (see Additional file 3: Table 
997 S6). The occurrence of the repeat variants indicated for coding sequences (CDS) correspond to

998 their occurrence as counted at the nucleotide level, whereas the occurrence in proteins is counted

999 at the amino acid sequence level (and may be higher due to different nucleotide sequences

1000 encoding the same amino acid sequence). The analysis of intraexonic repeat insertions is

1001 commented in more detail in Additional file 2: Note S4. 
bioRxiv preprint doi: https://doi.org/10.1101/2021.11.17.468966; this version posted November 19, 2021. The copyright holder for this preprint (which was not certified by peer review) is the author/funder, who has granted bioRxiv a license to display the preprint in perpetuity. It is made available under aCC-BY-NC-ND 4.0 International license.

(a)

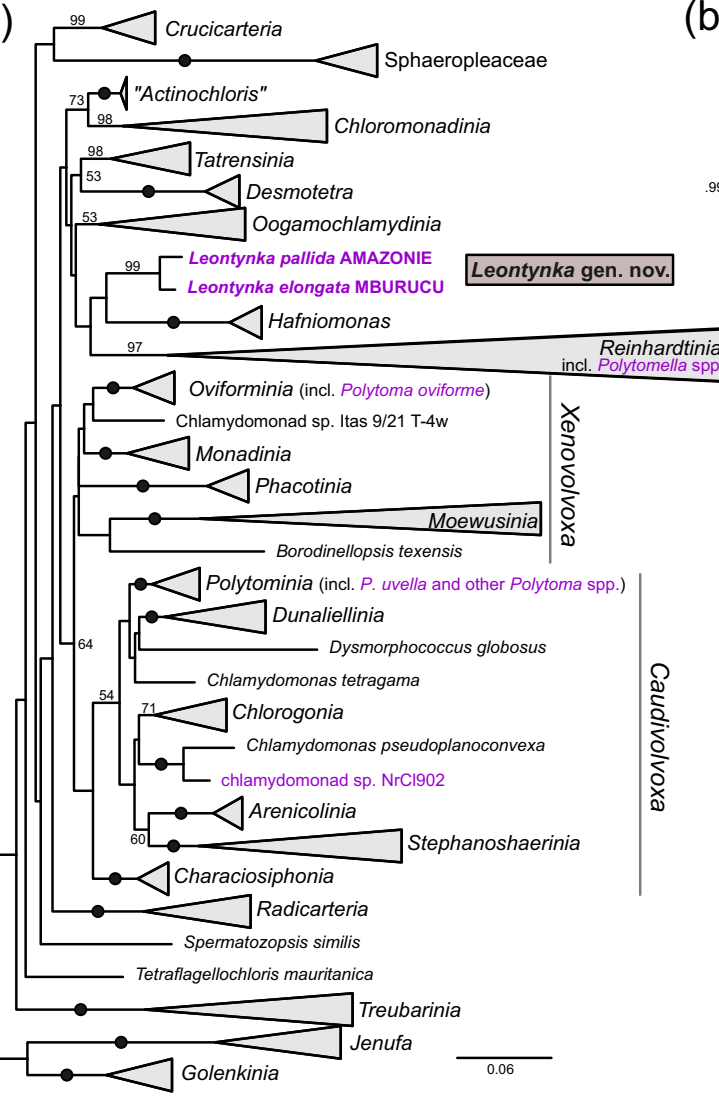

(b)

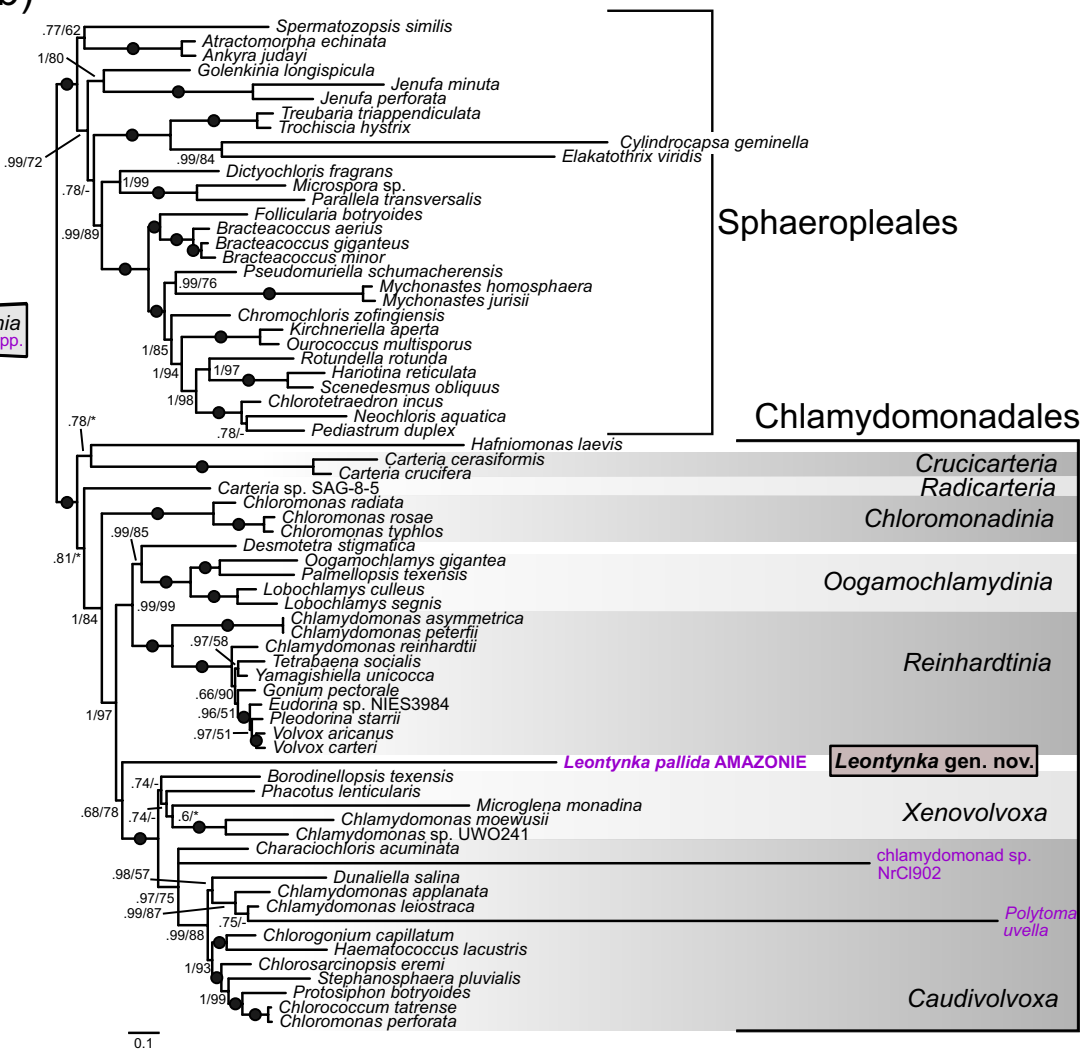




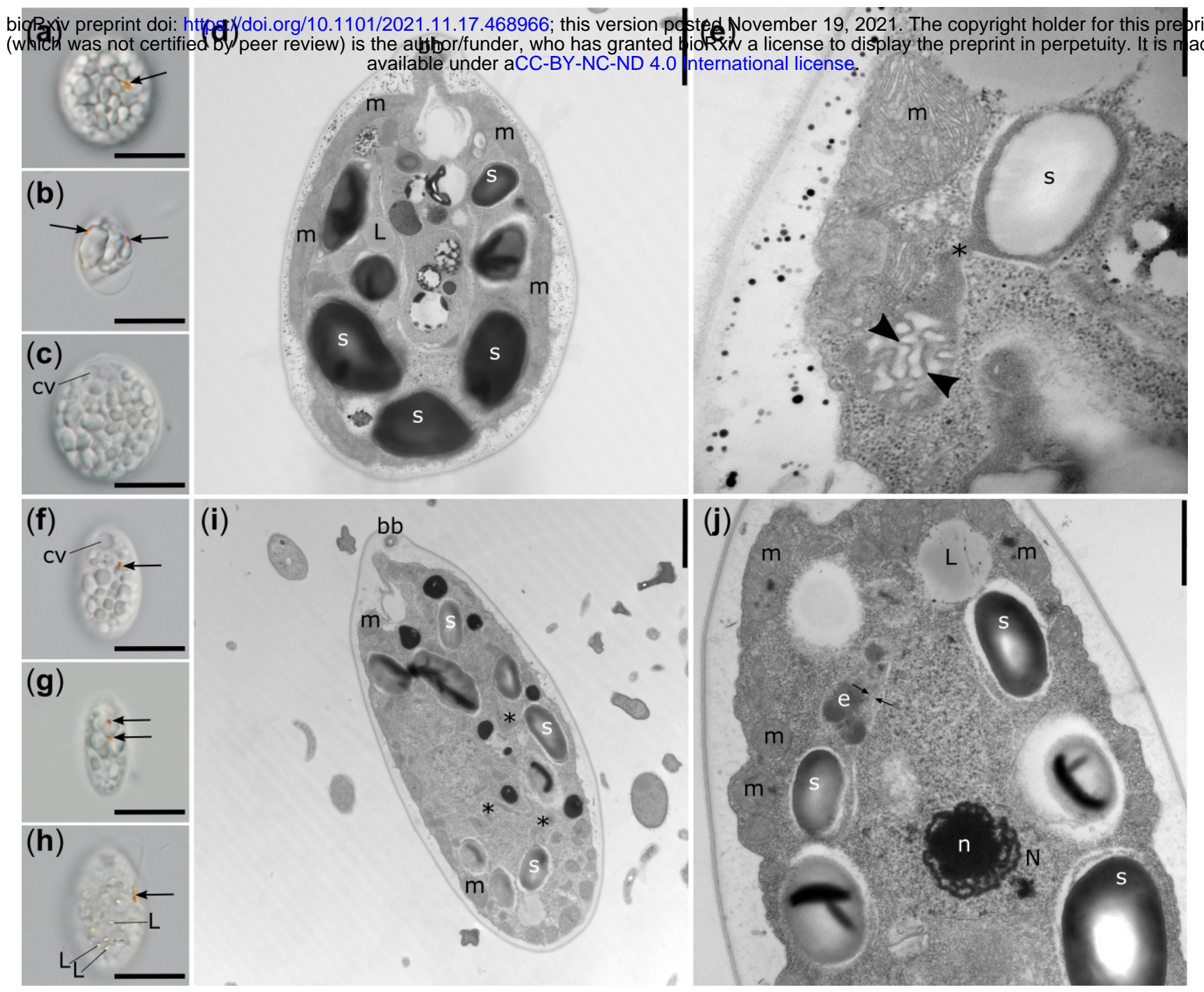


bioRxiv preprint doi: https://doi.org/10.1101/2021.11.17.468966; this version posted November 19,2021 . The copyright holder for this preprint (which was not certified by peer review) is the author/funder, who has granted bioRxiv a license to display the preprint in perpetuity. It is made available under aCC-BY-NC-ND 4.0 International license.

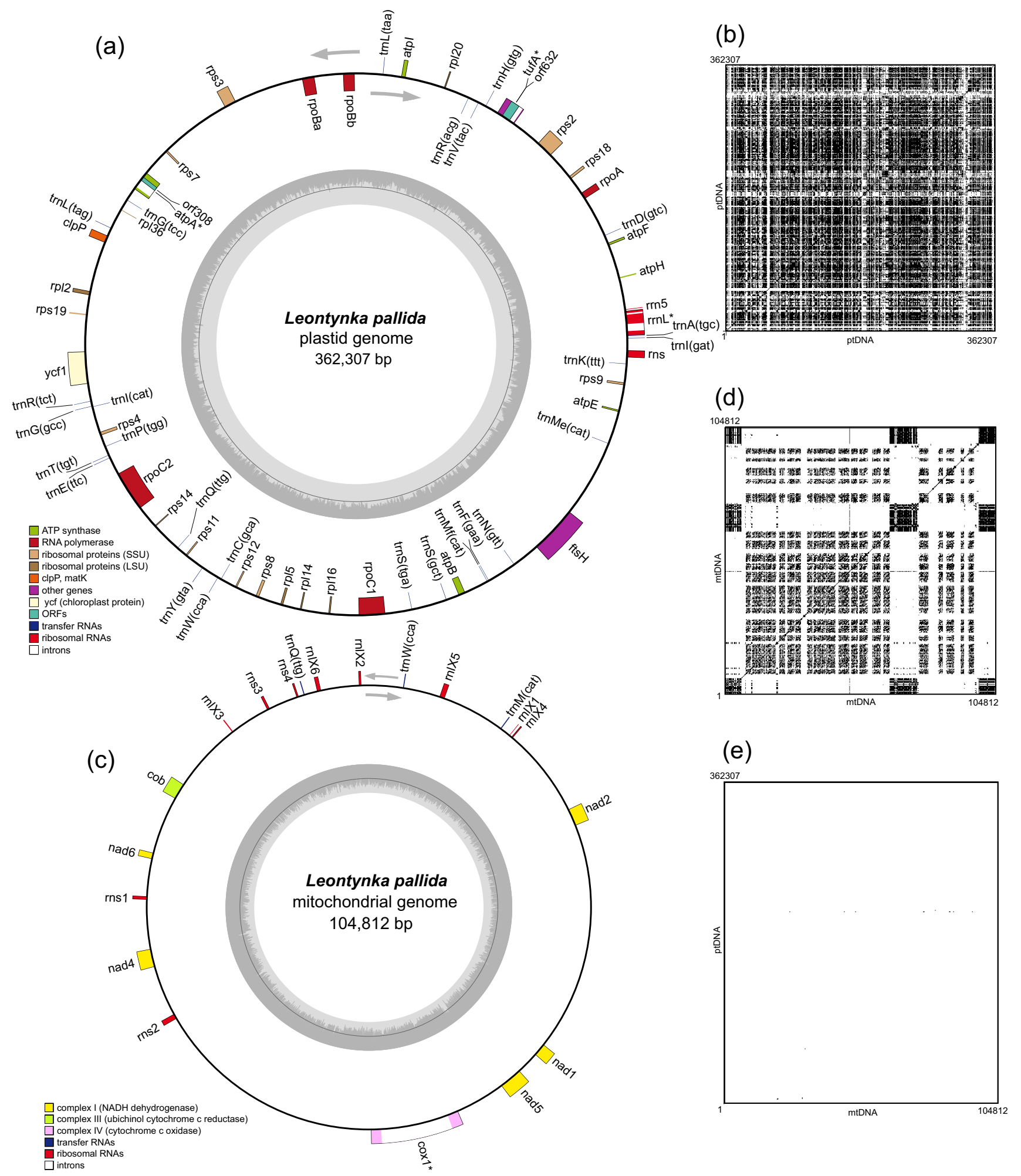


bioRxiv preprint doi: https://doi.org/10.1101/2021.11.17.468966; this version posted November 19, 2021. The copyright holder for this preprint (which was not certified by peer review) is the author/funder, who has granted bioRxiv a license to display the preprint in perpetuity. It is made available under aCC-BY-NC-ND 4.0 International license.

(a)

\begin{tabular}{|c|c|c|c|c|c|c|}
\hline Imperfect palindrome & $\begin{array}{c}\text { Typical } \\
\text { spacer }\end{array}$ & $\begin{array}{c}\text { spacer } \\
\text { lenght }\end{array}$ & Mismatch & \multicolumn{3}{|c|}{$\begin{array}{c}\text { Occurence } \\
\text { mtDNA ptDNA Exons }\end{array}$} \\
\hline CCCGCCCC.... GGGGCGGG & TTTC & $4-5$ & 0 & 119 & 0 & 0 \\
\hline (G)AACCGTTC .... GAACCGTT(C) & CCCC & $3-4$ & 1 & $96(80)$ & 0 & 0 \\
\hline CCAGCCCCG ...... CGGGGCGGG & CCCCTT & 7 & 1 & 57 & 0 & 0 \\
\hline (AC)AACATGTT ..... AACATGTT(CT) & CTCTT & 5 & $0(1)$ & $30(9)$ & 0 & 0 \\
\hline CAAACCAGT .. ACTGGTTAG & AA & $2-3$ & 1 & 0 & 1326 & 0 \\
\hline AAGCCAGC... GCTGACTT & AAA & 3 & 1 & 0 & 933 & 51 \\
\hline TATATTAGCT .. AGCTAATATA & AA & $1-5$ & 0 & 0 & 815 & 0 \\
\hline CTAGTGTTGCT . AGCAACACTAG & T & $0-3$ & 0 & 0 & 546 & 0 \\
\hline CTCGCTTGAACAT ATGTCAAGCGAG & - & 0 & 0 & 0 & 449 & 0 \\
\hline
\end{tabular}

(b)

\begin{tabular}{|c|c|c|c|c|c|c|c|c|c|c|c|}
\hline & atpl & $s H$ & rpoBa & rpoBb & rpo & rpoC2 & $r p s 2$ & $r p s 3$ & $r p s 8$ & $r p s 9$ & $v C$ \\
\hline \multirow{2}{*}{\begin{tabular}{|l} 
IP (typical) \\
IP (general)
\end{tabular}} & $0 / 0$ & \begin{tabular}{|l|l|}
$0 / 4$ \\
\end{tabular} & $0 / 0$ & $2 / 0$ & $4 / 1$ & $3 / 0$ & $2 / 0$ & $2 / 0$ & $0 / 1$ & $0 / 0$ & \\
\hline & $0 / 0$ & $0 / 7$ & $0 / 0$ & $5 / 0$ & $7 / 1$ & $15 / 0$ & $2 / 0$ & $6 / 0$ & $0 / 1$ & $0 / 0$ & $7 / 0$ \\
\hline variant 1 & 1 & 15 & 2 & 1 & 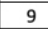 & 22 & 4 & 1 & 0 & 0 & 4 \\
\hline an & \begin{tabular}{|l|}
$0 / 0$ \\
\end{tabular} & $1 / 4$ & $0 / 0$ & $2 / 0$ & $5 / 1$ & $/ 1$ & $2 / 0$ & $5 / 0$ & $1 / 1$ & $0 / 0$ & $7 / 0$ \\
\hline aria & \begin{tabular}{|l|}
$0 / 0$ \\
\end{tabular} & $4 / 1$ & $0 / c$ & $0 / 1$ & $4 / c$ & $1 / 0$ & $1 / 1$ & $1 / 0$ & $0 / 0$ & $1 / 0$ & $1 / 0$ \\
\hline gariant 4 & \begin{tabular}{|l|}
$0 / 0$ \\
\end{tabular} & $0 / 0$ & $0 / 6$ & $2 / 0$ & $3 / 1$ & $3 / 0$ & $2 / 0$ & $2 / 0$ & D/1 & $0 / 0$ & $6 / 0$ \\
\hline ariant 5 & \begin{tabular}{|l|}
$1 / 0$ \\
\end{tabular} & $10 / 0$ & $0 / 2$ & $0 / 0$ & $3 / 0$ & $14 / 1$ & $1 / 0$ & $0 / 0$ & $0 / 0$ & $0 / 0$ & $1 / 0$ \\
\hline variant 6 & \begin{tabular}{|l|}
$1 / 0$ \\
\end{tabular} & $11 / 1$ & $0 / 2$ & $1 / 0$ & $5 / 5$ & $17 / 3$ & $2 / 0$ & $0 / 1$ & $0 / 0$ & $0 / 0$ & $2 / 1$ \\
\hline variant 7 & $1 / 0$ & $12 / 4$ & $0 / 3$ & $5 / 0$ & $9 / 2$ & $31 / 1$ & $4 / 1$ & $7 / 0$ & $0 / 1$ & $0 / 0$ & $10 / 0$ \\
\hline & & & & & & & $5 / 1$ & $3 / 2$ & $1 / 1$ & 0/0 & \\
\hline
\end{tabular}

\begin{tabular}{|c|c|c|c|c|c|c|c|c|c|c|c|c|c|c|}
\hline \multirow{2}{*}{ (c) } & & & & & & & & & & \multicolumn{4}{|c|}{ Leontynka pallida AMAZONIE } & \multirow{3}{*}{$\begin{array}{c}\text { Other ptDNAs } \\
0 / 0\end{array}$} \\
\hline & & & & & & & & & & exons $(+/-)$ & introns ( $+/-)$ & ptDNA (+/-) & mtDNA $(+/-)$ & \\
\hline \multirow{2}{*}{\multicolumn{3}{|c|}{$\begin{array}{l}\text { IP (typical) } \\
\text { IP (general) }\end{array}$}} & AAG & CCA & GCA & AAG & CTG & ACT & $\mathrm{T}$ & $20 / 6$ & $2 / 0$ & $262 / 254$ & $0 / 0$ & \\
\hline & & & AAG & CCA & GCN & NNG & CTG & ACT & $\mathrm{T}$ & $42 / 9$ & $3 / 0$ & $457 / 476$ & $0 / 0$ & $0 / 0$ \\
\hline \multirow{3}{*}{\multicolumn{3}{|c|}{$\begin{array}{l}\text { variant } 1 \\
\text { variant } 2 \\
\text { variant } 3\end{array}$}} & AAG & TCA & GCN & NNG & CTG & ACT & $T$ & 59 & 1 & 142 & 0 & 0 \\
\hline & & & $\mathrm{AA}[\mathrm{AG}]$ & CCA & GCA & AA[ACG] & $\mathrm{C}[\mathrm{CT}][\mathrm{TG}]$ & $\mathrm{AC}[\mathrm{AT}]$ & $\mathrm{T}$ & $27 / 7$ & $2 / 0$ & $270 / 261$ & $0 / 0$ & $0 / 0$ \\
\hline & & & $\mathrm{AA}[\mathrm{AG}]$ & TCA & GCA & AA[ACG] & $\mathrm{C}[\mathrm{CT}][\mathrm{TG}]$ & $\mathrm{AC}[\mathrm{AT}]$ & $\mathrm{T}$ & $13 / 3$ & $0 / 0$ & $15 / 13$ & $0 / 0$ & $0 / 0$ \\
\hline variant 4 & AAA & GAT & AAG & CCA & GCA & AAG & CTG & ACT & TC & $18 / 2$ & $2 / 0$ & $187 / 196$ & $0 / 0$ & $0 / 0$ \\
\hline variant 5 & AAA & GAT & AAG & TCA & GCA & GAG & CTG & ACT & TC & $30 / 3$ & $0 / 1$ & $40 / 52$ & $0 / 0$ & $0 / 0$ \\
\hline variant 6 & AAA & GAT & AAG & TCA & GCN & NNG & CTG & $\mathrm{AC}[\mathrm{AT}]$ & TC & $39 / 13$ & $0 / 1$ & $58 / 67$ & $0 / 0$ & $0 / 0$ \\
\hline variant 7 & AAA & GAT & AAG & {$[\mathrm{CT}] \mathrm{CA}$} & GCA & NNG & $\mathrm{CT}[\mathrm{TG}]$ & ACT & TC & $79 / 12$ & $3 / 1$ & $428 / 480$ & $0 / 0$ & $0 / 0$ \\
\hline variant 8 & AAA & GAT & $\mathrm{AA}[\mathrm{AG}]$ & {$[\mathrm{CT}][\mathrm{CT}] \mathrm{A}$} & GC[AGT] & NNN & {$[\mathrm{CT}] \mathrm{T}[\mathrm{TGA}]$} & ACT & TC & $93 / 22$ & $3 / 1$ & $444 / 502$ & $0 / 0$ & $0 / 0$ \\
\hline
\end{tabular}

(e)

\begin{tabular}{|c|c|c|c|c|c|c|c|c|c|c|}
\hline \multicolumn{3}{|c|}{ (e) } & \multicolumn{5}{|c|}{ codon usage } & & \multicolumn{2}{|c|}{ occurence } \\
\hline RF & nucleotide sequence & AA sequence & rare $2 \%$ & rare $4 \%$ & rare $10 \%$ & rare $\mathbf{2 0 \%}$ & frequent $50 \%$ & Rare AA & $\operatorname{CDS}$ & proteins \\
\hline+0 & AAAGATAAGCCAGCAAAGCTGACTTCN & KDKPAKLTS & $0(\max 1)^{*}$ & $0(\max 1)^{*}$ & $1(\max 2)$ & $1(\max 2)$ & $5(\max 6)$ & 0 & 18 & 22 \\
\hline+1 & NAAAGATAAGCCAGCAAAGCTGACTTC/N & XR*ASKADF & - & - & - & - & - & - & - & - \\
\hline+2 & NNAAAGATAAGCCAGCAAAGCTGACTT/CN & XKISQQS*L & - & - & - & - & - & - & - & - \\
\hline-0 & GAAGTCAGCTTTGCTGGCTTATCTTTN & EVSFAGLSX & 0 & $0(\max 1)^{*}$ & $2(\max 3)$ & $3(\max 4)$ & $5(\max 6)$ & 0 & 2 & 6 \\
\hline-1 & NGAAGTCAGCTTTGCTGGCTTATCTTT/N & XSQLCWLIF & $1(\max 2)$ & $1(\max 2)$ & $2(\max 3)$ & $4(\max 5)$ & 1 & 2 & 0 & 0 \\
\hline-2 & NNGAAGTCAGCTTTGCTGGCTTATCTT/TN & XKSALLAYL & $0(\max 1)$ & $1(\max 2)$ & $2(\max 3)$ & $2(\max 3)$ & $3(\max 4)$ & 0 & 0 & 0 \\
\hline
\end{tabular}

(d)

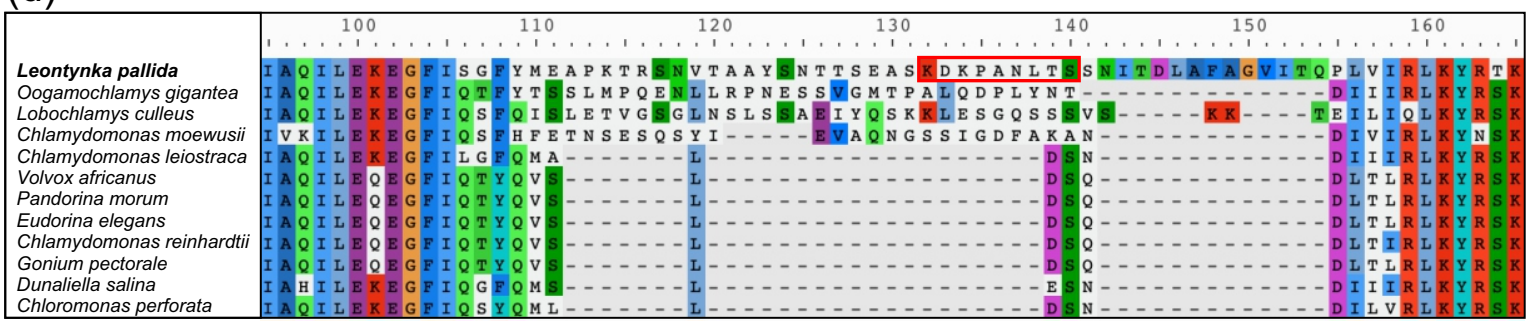

